Resource.Assessment

New gersey

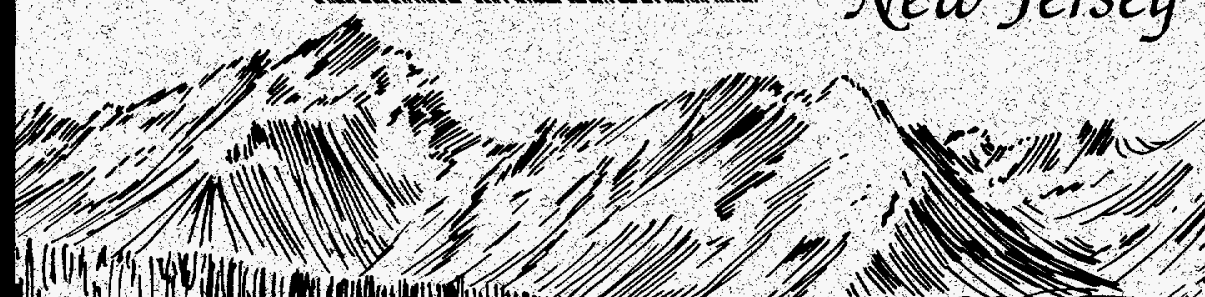

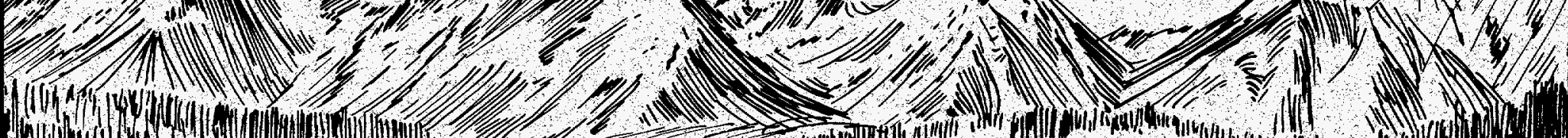
(n)

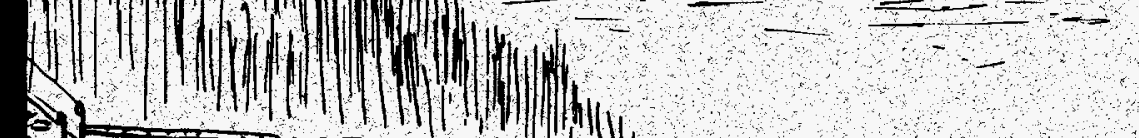

1 $\longrightarrow$

.

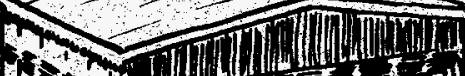
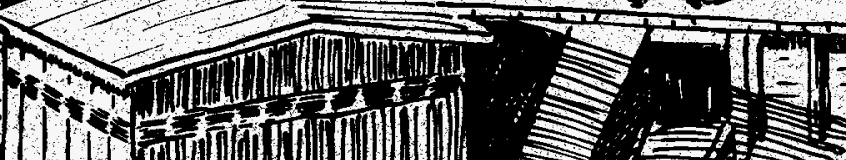

(1)
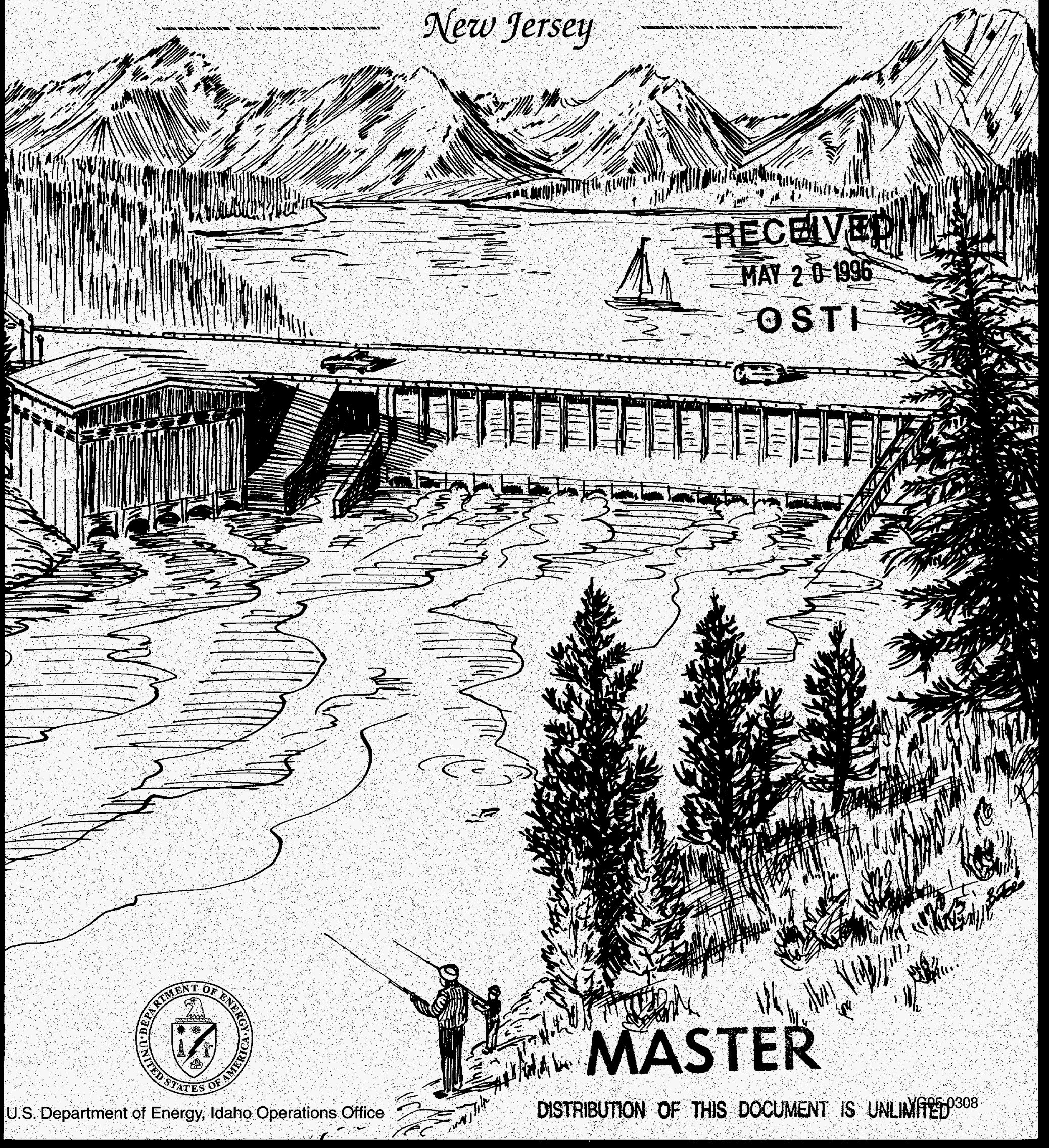

DOE/ID-10430(NJ)

\title{
U.S. Hydropower Resource Assessment for New Jersey
}

\author{
Prepared by: \\ Alison M. Conner \\ James E. Francfort \\ Project Manager: \\ Ben N. Rinehart \\ Published March 1996 \\ Idaho Falls, Idaho 83415
}

Idaho National Engineering Laboratory Renewable Energy Products Department Lockheed Idaho Technologies Company

Prepared for the

U.S. Department of Energy

Assistant Secretary for Energy Efficiency and Renewable Energy Under DOE Idaho Operations Office

Contract DE-AC07-941D13223 



\begin{abstract}
The U.S. Department of Energy is developing an estimate of the undeveloped hydropower potential in the United States. The Hydropower Evaluation Software (HES) is a computer model that was developed by the Idaho National Engineering Laboratory for this purpose. HES measures the undeveloped hydropower resources available in the United States, using uniform criteria for measurement. The software was developed and tested using hydropower information and data provided by the Southwestern Power Administration. It is a menu-driven program that allows the personal computer user to assign environmental attributes to potential hydropower sites, calculate development suitability factors for each site based on the environmental attributes present, and generate reports based on these suitability factors. This report describes the resource assessment results for the State of New Jersey.
\end{abstract}

\title{
DISCLAIMER
}

This report was prepared as an account of work sponsored by an agency of the United States Government. Neither the United States Government nor any agency thereof, nor any of their employees, makes any warranty, express or implied, or assumes any legal liability or responsibility for the accuracy, completeness, or usefulness of any information, apparatus, product, or process disclosed, or represents that its use would not infringe privately owned rights. Reference herein to any specific commercial product, process, or service by trade name, trademark, manufacturer, or otherwise does not necessarily constitute or imply its endorsement, recommendation, or favoring by the United States Government or any agency thereof. The views and opinions of authors expressed herein do not necessarily state or reflect those of the United States Government or any agency thereof. 


\section{ACKNOWLEDGMENTS}

The authors thank Peggy A. M. Brookshier, John V. Flynn, and Wayne Belgrave of the U.S. Department of Energy, and William Crom of the State of New Jersey for their active participation and timely comments. 


\section{CONTENTS}

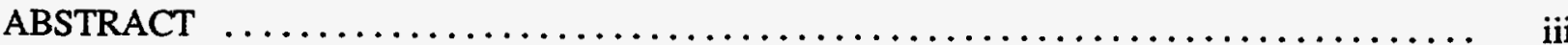

ACKNOWLEDGMENTS $\ldots \ldots \ldots \ldots \ldots \ldots \ldots \ldots \ldots \ldots \ldots \ldots \ldots \ldots \ldots \ldots \ldots$ vii

INTRODUCTION $\ldots \ldots \ldots \ldots \ldots \ldots \ldots \ldots \ldots \ldots \ldots \ldots \ldots \ldots \ldots \ldots \ldots \ldots \ldots$

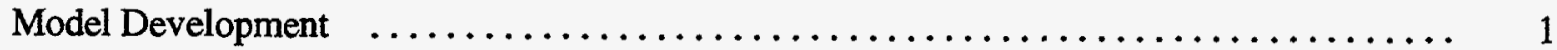

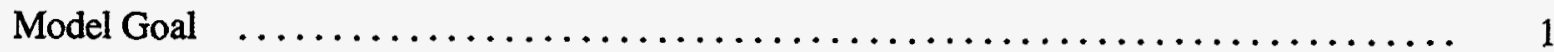

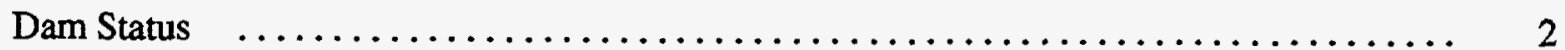

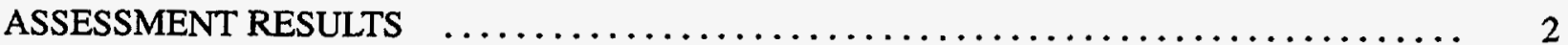

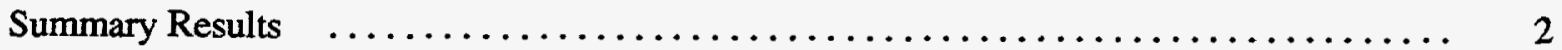

Detailed Results $\quad \ldots \ldots \ldots \ldots \ldots \ldots \ldots \ldots \ldots \ldots \ldots \ldots \ldots \ldots \ldots \ldots \ldots \ldots \ldots$

OBTAINING INDIVIDUAL STATE INFORMATION $\ldots \ldots \ldots \ldots \ldots \ldots \ldots \ldots \ldots \ldots \ldots$

ADDITIONAL HYDROPOWER EVALUATION SOFTWARE INFORMATION $\ldots \ldots \ldots \ldots$

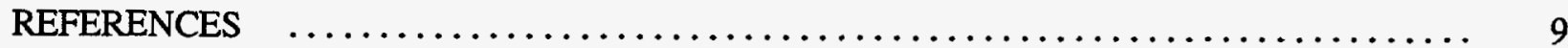

APPENDIX A-Summary Report $\ldots \ldots \ldots \ldots \ldots \ldots \ldots \ldots \ldots \ldots \ldots \ldots \ldots \ldots \ldots \ldots \ldots \ldots$

APPENDIX B-River Basins Report $\ldots \ldots \ldots \ldots \ldots \ldots \ldots \ldots \ldots \ldots \ldots \ldots \ldots \ldots \ldots \ldots \ldots \ldots$

APPENDIX C-New Jersey Sites Listing $\ldots \ldots \ldots \ldots \ldots \ldots \ldots \ldots \ldots \ldots \ldots \ldots \ldots \ldots$

APPENDIX D-Individual Resource Database Listing $\ldots \ldots \ldots \ldots \ldots \ldots \ldots \ldots \ldots \ldots$ D-1

APPENDIX E-List of 469 Small Sites $\ldots \ldots \ldots \ldots \ldots \ldots \ldots \ldots \ldots \ldots \ldots \ldots \ldots \ldots \ldots \ldots$

\section{FIGURES}

1. Number of sites, by capacity groups, with HES-modeled undeveloped hydropower potential

2. The nonmodeled and HES-modeled undeveloped hydropower potential $\ldots \ldots \ldots \ldots \ldots$

3. The number of sites with undeveloped hydropower potential and the total megawatts of HES-modeled undeveloped hydropower potential

4. The Great Falls plant is an example of a developed New Jersey hydropower plant in an urban setting

5. Number of sites with undeveloped hydropower potential in the

New Jersey river basins 
6. Megawatts of HES-modeled undeveloped hydropower potential in the

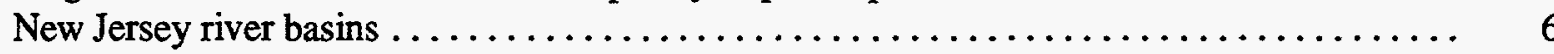

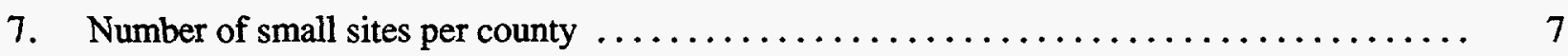

8. Nonmodeled hydropower potential capacity per county for the small sites $\ldots \ldots \ldots \ldots \ldots$

\section{TABLES}

1. Undeveloped hydropower potential summaries for New Jersey $\ldots \ldots \ldots \ldots \ldots \ldots \ldots \ldots$ 


\section{U.S. Hydropower Resource Assessment for New Jersey}

\section{INTRODUCTION}

In June 1989, the U.S. Department of Energy initiated the development of a National Energy Strategy to identify the energy resources available to support the expanding demand for energy in the United States. Public hearings conducted as part of the strategy development process indicated that undeveloped hydropower resources were not well defined. As a result, the Department of Energy established an interagency Hydropower Resource Assessment Team to ascertain the undeveloped hydropower potential. In connection with these efforts by the Department of Energy, the Idaho National Engineering Laboratory designed the Hydropower Evaluation Software (HES), which has been used to perform a resource assessment of the undeveloped conventional hydropower potential in over 30 states. This report presents the results of the hydropower resource assessment for the State of New Jersey. Undeveloped pumped storage hydropower potential is not included.

The HES was developed as a tool to measure undeveloped hydropower potential regionally or by state. The software is not intended to provide precise development factors for individual sites, but to provide regional or state totals. Because the software was developed as a generic measurement tool encompassing national issues, regional and state totals must be considered judiciously; various local issues may skew undeveloped hydropower potential totals. The information for the resource assessment was compiled from the Federal Energy Regulatory Commission's Hydroelectric Power Resources Assessment database and several other sources. Refer to DOE/ID-10338, the User's Manual (Francfort, Matthews, Rinehart 1991) for the specifics of the software and to DOE/ID-10430.1, the Status Report (Conner, Francfort, Rinehart 1996) for an overview of all resource assessment activities to date.

\section{Model Development}

Hydropower Evaluation Software, both a probability-factor computer model and a database, is a menu-driven program that is intended to be user-friendly. Computer screens and report-generation capabilities were developed to meet the needs of users nationwide. The software uses environmental attribute data to generate an overall project environmental suitability factor (PESF) between 0.1 and 0.9 , where 0.9 indicates the highest likelihood of development and 0.1 indicates the lowest likelihood of development. The suitability factors are dependant on the unique environmental attributes of each potential site. They reflect the considerations that (a) environmental concerns can make a potential site unacceptable, prohibiting its development (for a suitability factor of 0.1 ), or (b) if there are no environmental concerns, there is no effect on the likelihood of site development (for a suitability factor of 0.9). A combination of attributes can result in a lower suitability factor because multiple environmental considerations would reduce the likelihood that a site may be developed to its physical potential.

\section{Model Goal}

The goal of the HES is to assemble an accurate resource database of all sites with undeveloped hydropower potential in the United States for use as a planning tool to determine the viable national hydropower potential. Undeveloped hydropower potential is not limited to the development of new sites; it also includes the development of additional hydropower-generating capacity at sites that currently have hydropower, but are not developed to their full potential. This undeveloped hydropower potential is a source of nonpolluting, renewable energy available to meet the growing 
power needs of the United States. The HES should help make this goal obtainable and ensure a set of uniform criteria for national assessment.

\section{Dam Status}

The effects of environmental attributes vary by dam status. The dam status classifications used are as follows

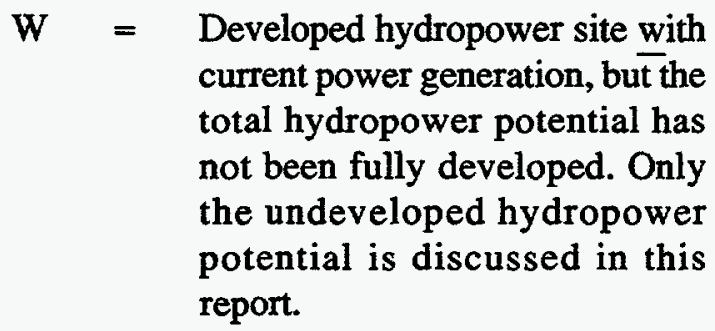

$\mathrm{W} / \mathrm{O}=$ Developed site without current power generation. The site has some type of developed impoundment or diversion structure, but no developed hydropower generating capability.

$\mathrm{U}=$ Undeveloped site. The site does not have power generation capability nor a developed impoundment or diversion structure.

\section{ASSESSMENT RESULTS}

\section{Summary Results}

A total of 12 sites (Table 1) have been identified and assessed for their undeveloped hydropower potential. The HES results for individual site capacities range from 23 kilowatts $(\mathrm{kW})$ to 4 megawatts (MW). Most sites (83\%) have potential capacities of under $1 \mathrm{MW}$ (Figure 1).

The nonmodeled undeveloped hydropower potential total for New Jersey was identified as $11 \mathrm{MW}$. The HES results lowers this estimate about $18 \%$ to $9 \mathrm{MW}$. New Jersey did not report any sites with undeveloped hydropower potential at sites with current generating capabilities. Overall, the HES-modeled results did not significantly change the capacity in undeveloped hydropower potential (Figure 2). The number of sites does not change, only the identified undeveloped hydropower potential is reassessed (Figure 3).

The 12 identified sites are located within 1 major river basin and 9 minor river basins. The major river basin, the Delaware, contains 3 hydropower sites, and the remaining 9 sites are located in minor river basins (Figure 5). The majority of the undeveloped hydropower potential $(97 \%)$ in New Jersey is located in the minor river basins (Figure 6).

Table 1. Undeveloped hydropower potential summaries for New Jersey. The table contains the nonmodeled undeveloped name plate potential, as well as the HES-modeled undeveloped hydropower potential totals.

\begin{tabular}{lccc}
\hline & Number of projects & $\begin{array}{c}\text { Name plate potential } \\
(\mathrm{MW})\end{array}$ & $\begin{array}{c}\text { HES-modeled potential } \\
(\mathrm{MW})\end{array}$ \\
\cline { 2 - 4 } With Power & 0 & 0 & 0 \\
W/O Power & 9 & 6.1 & 5.3 \\
Undeveloped & 3 & 4.6 & 4.1 \\
State Total & 12 & 10.7 & 9.4 \\
\hline
\end{tabular}




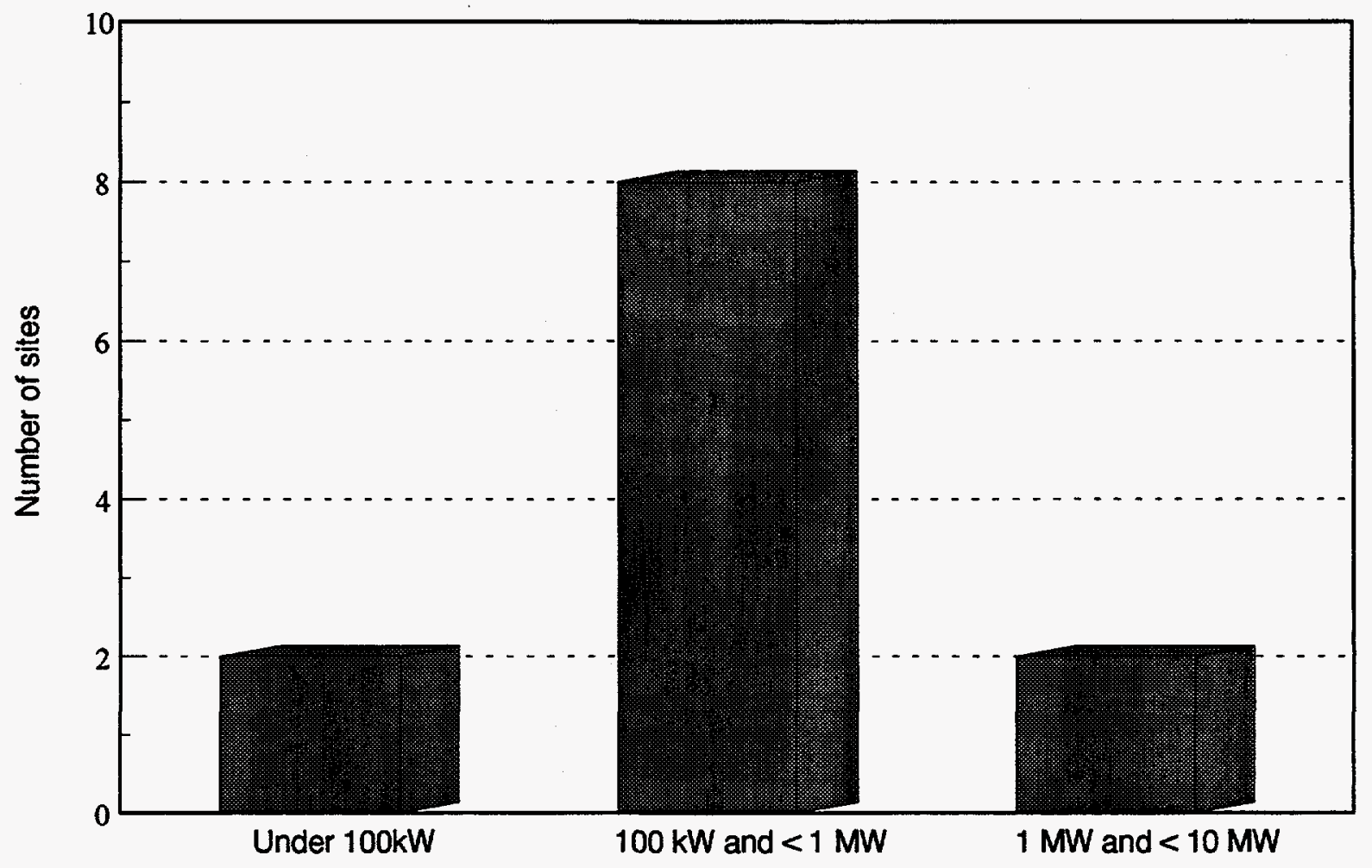

Figure 1. Number of sites, by capacity groups, with HES-modeled undeveloped hydropower potential.

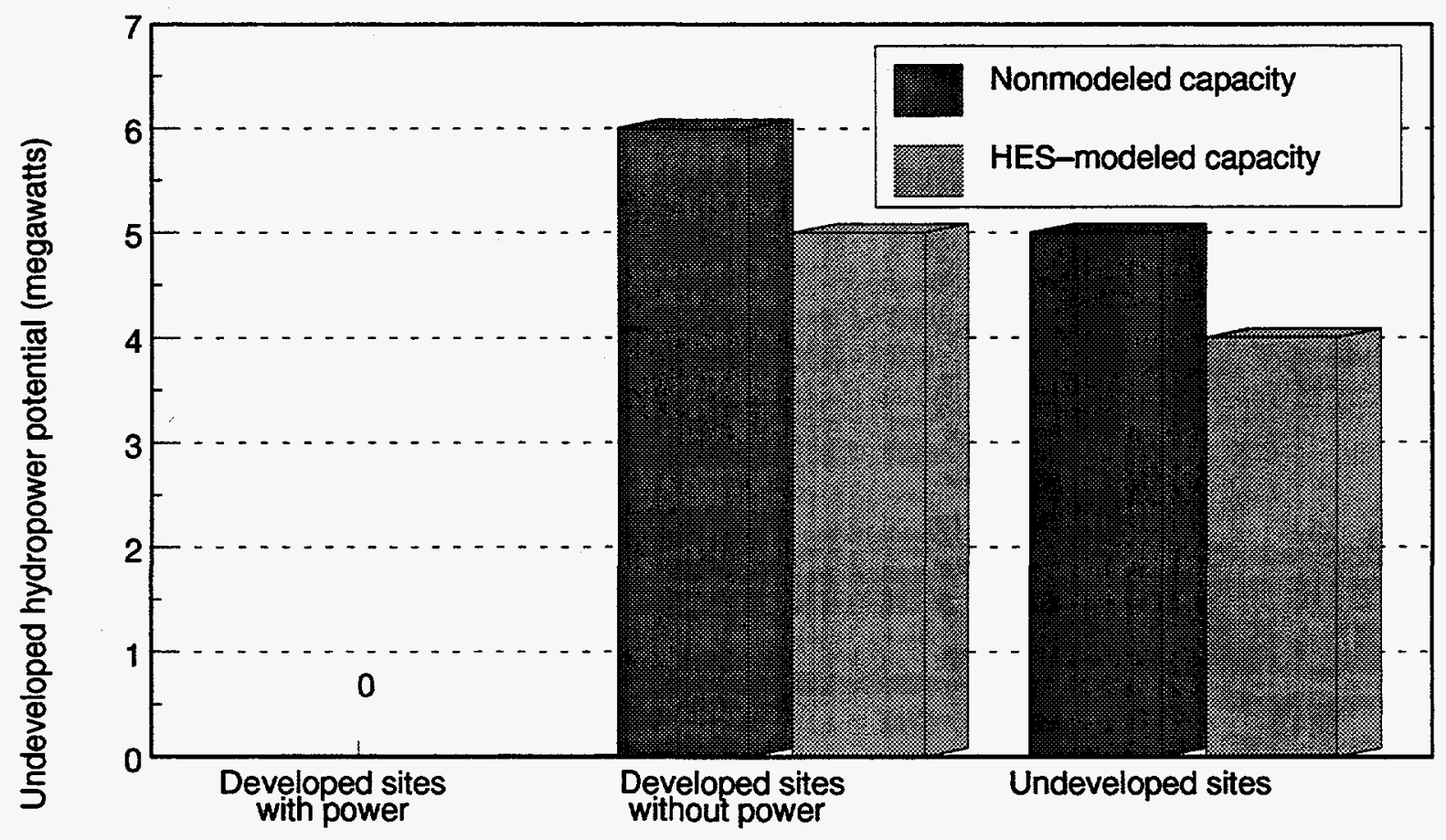

Figure 2. The nonmodeled and HES-modeled undeveloped hydropower potential. 


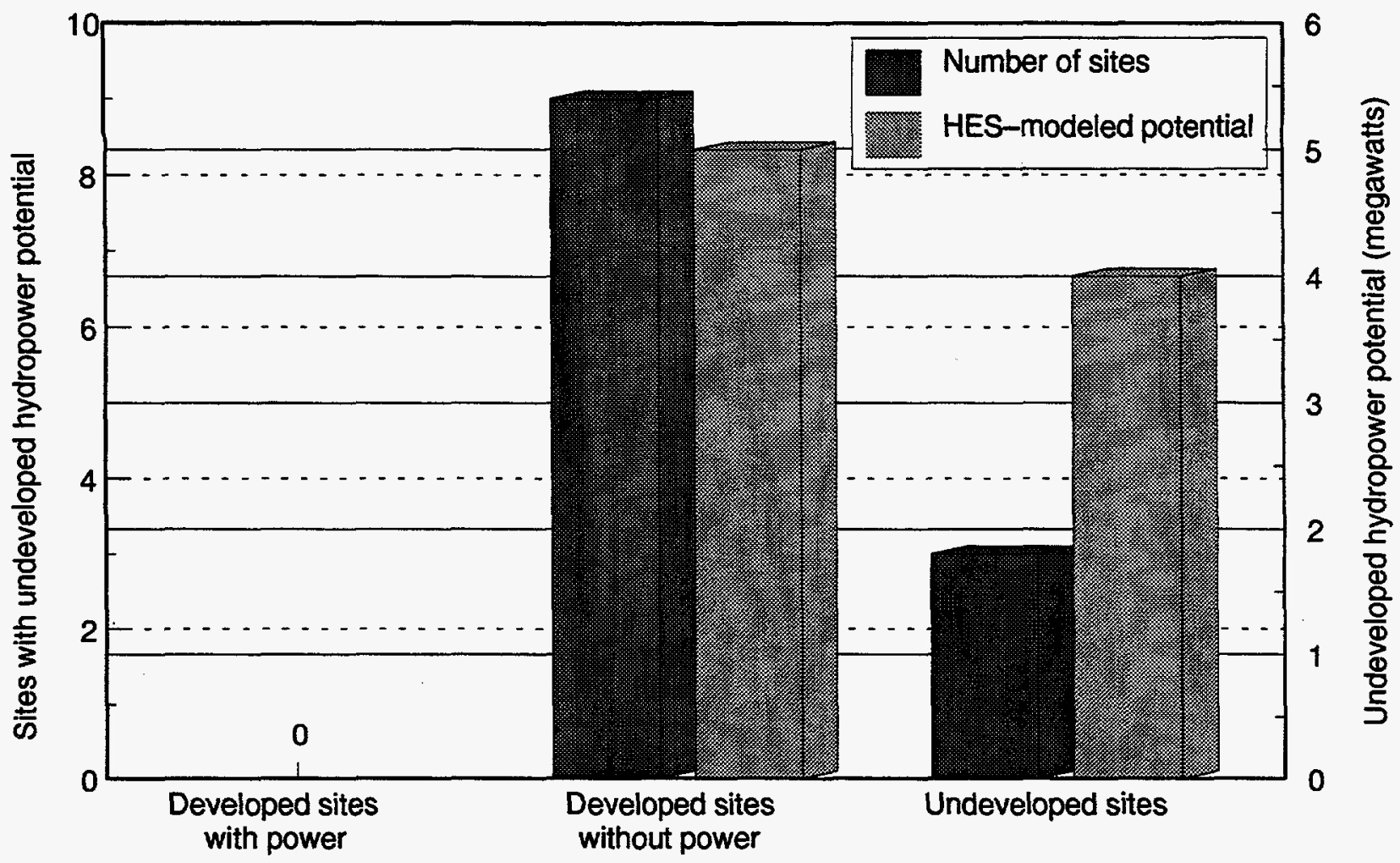

Figure 3. The number of sites with undeveloped hydropower potential and the total megawatts of HESmodeled undeveloped hydropower potential.

The State of New Jersey also provided information on 469 small hydropower sites with a total of $22 \mathrm{MW}$. Due to their relatively small size (average potential capacity of $47 \mathrm{~kW}$ per site) and limited commercial value, they were not included in the model. Figure 7 illustrates the total number of these small sites per county. Figure 8 identifies the total unmodeled megawatts of hydropower potential capacity per county for the small sites.

\section{Detailed Results}

The appendices contain, in the form of HESgenerated reports, detailed information about the undeveloped hydropower potential in New Jersey. The appendices contain the following information:

Appendix A The undeveloped hydropower potential is summarized by dam status groups. The number of sites, nonmodeled undeveloped hydropower potential, and HES- modeled undeveloped hydropower potential is provided based on the dam status.

Appendix B The hydropower resource assessment by river basin includes the project number, project name, stream name, dam status, nonmodeled undeveloped hydropower potential, and the HES-modeled undeveloped hydropower potential for each of the individual sites. Subtotals are provided for each river basin.

Appendix C This is a listing of the project numbers, plant name, stream name, if a site is Federally owned, nonmodeled undeveloped hydropower potential, and HES-modeled undeveloped hydropower potential. The sites are grouped by dam status. 


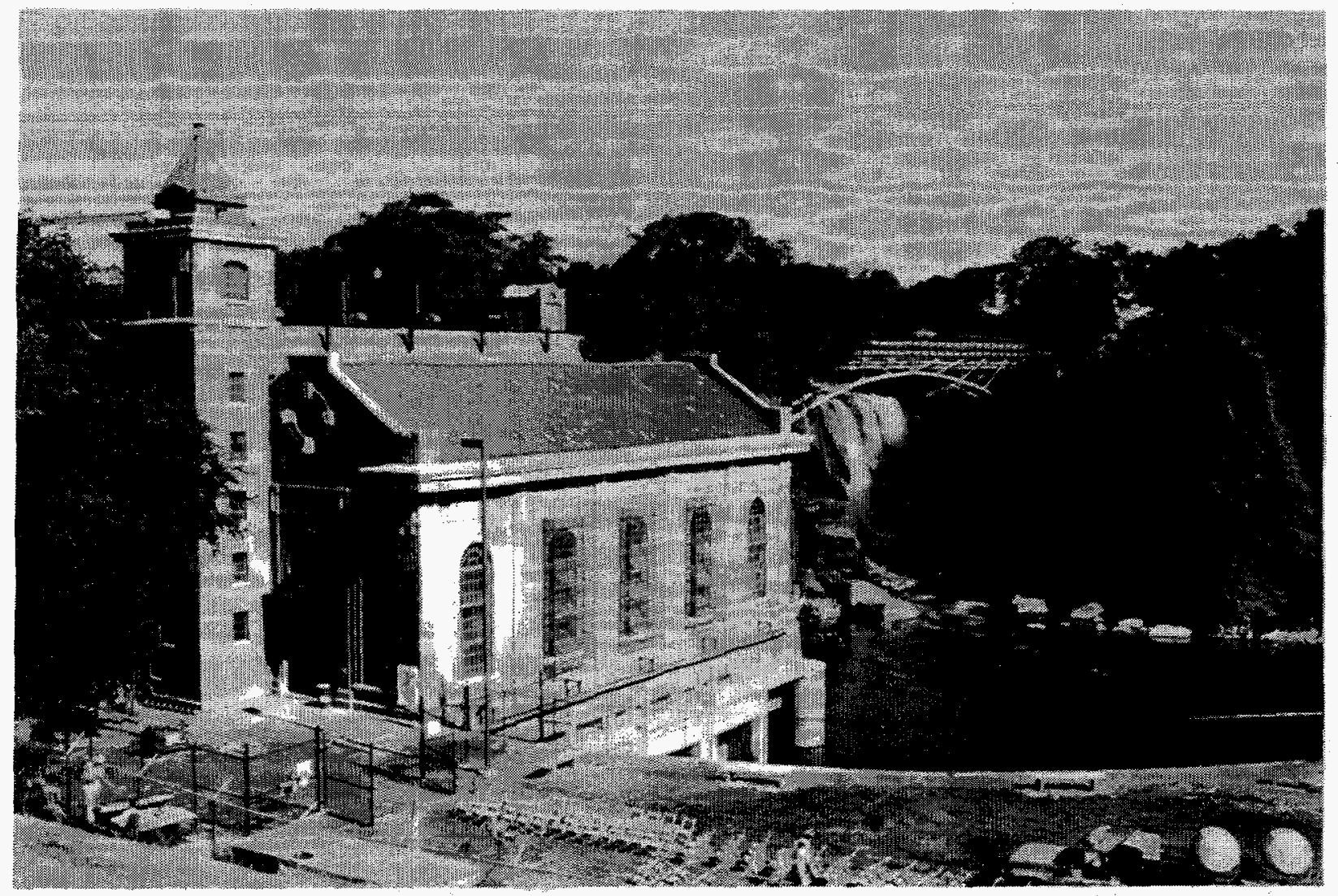

Figure 4. The Great Falls plant is an example of a developed New Jersey hydropower plant in an urban setting. The plant is the large brick building in the left center of the picture above. The Great Falls waterfall is located in the background, under the pedestrian and water piping bridges. The Great Falls power plant is a National Historic Landmark, located on the Passaic River, in Paterson, New Jersey. The plant, which has a capacity of $10.95 \mathrm{MW}$ and a head of 67 feet, was initially placed into service in 1914. It received a major upgrading during the 1980 s. Parts of the diversion dam and water conveyance system date to the 1840 s.

Appendix D This section contains a resource database list for each of the 149 sites in New Jersey. Information includes plant name, stream, state, county, river basin and owner names, project number, name plate and HES-modeled undeveloped hydropower potential, the unit and plant types, dam status, latitude, longitude, and the environmental factors that the HES uses to determine the project environmental suitability factor.
Appendix E This appendix lists 469 small sites (average capacity of $47 \mathrm{~kW}$ per site) with a total hydropower potential capacity of $22 \mathrm{MW}$. The list is sorted on county name and river.

\section{OBTAINING INDIVIDUAL STATE INFORMATION}

Additional copies of the hydropower resource assessment results for individual states are available and can be obtained by writing or calling the authors or the National Technical Information Service (NTIS). 


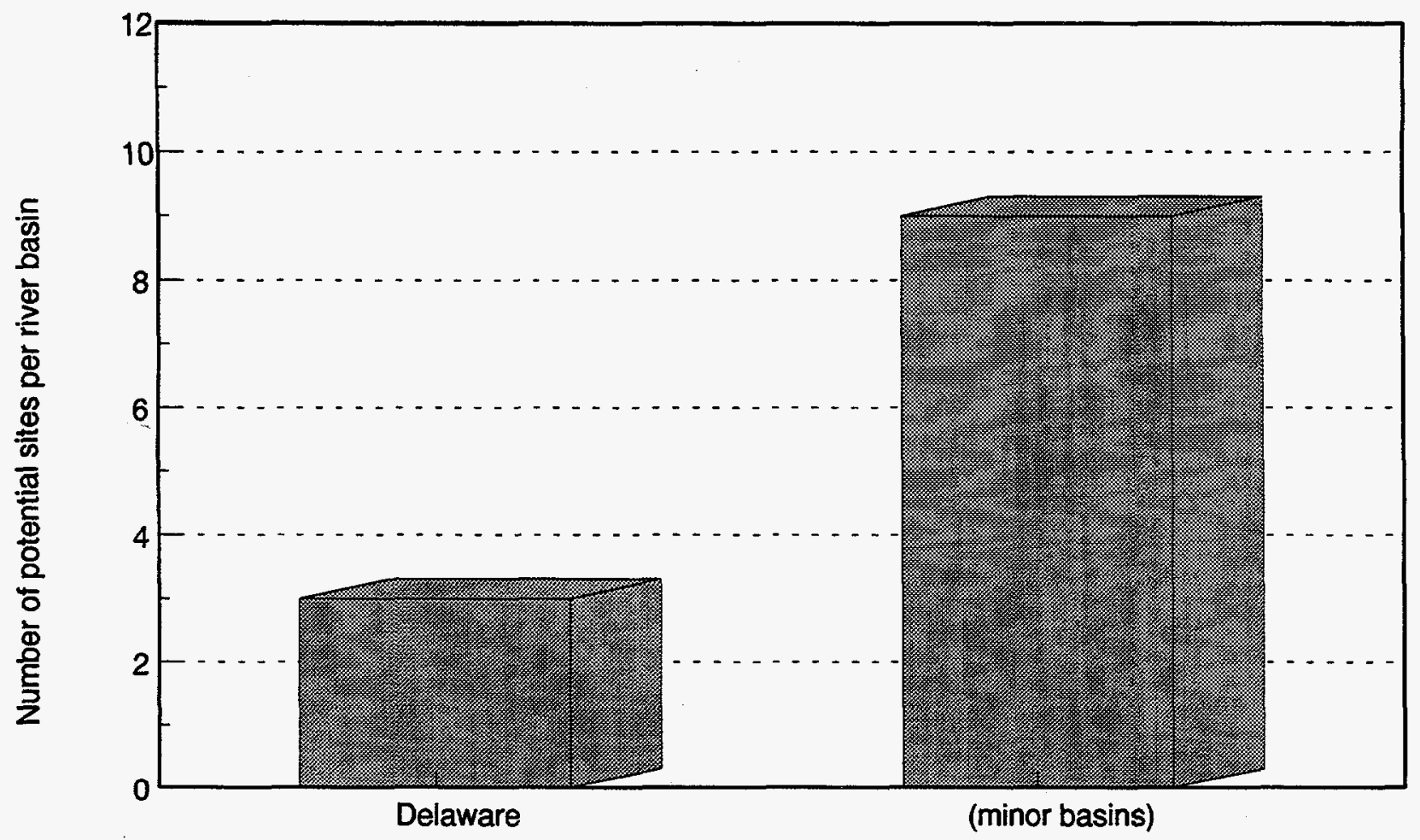

Figure 5. Number of sites with undeveloped hydropower potential in the New Jersey river basins.

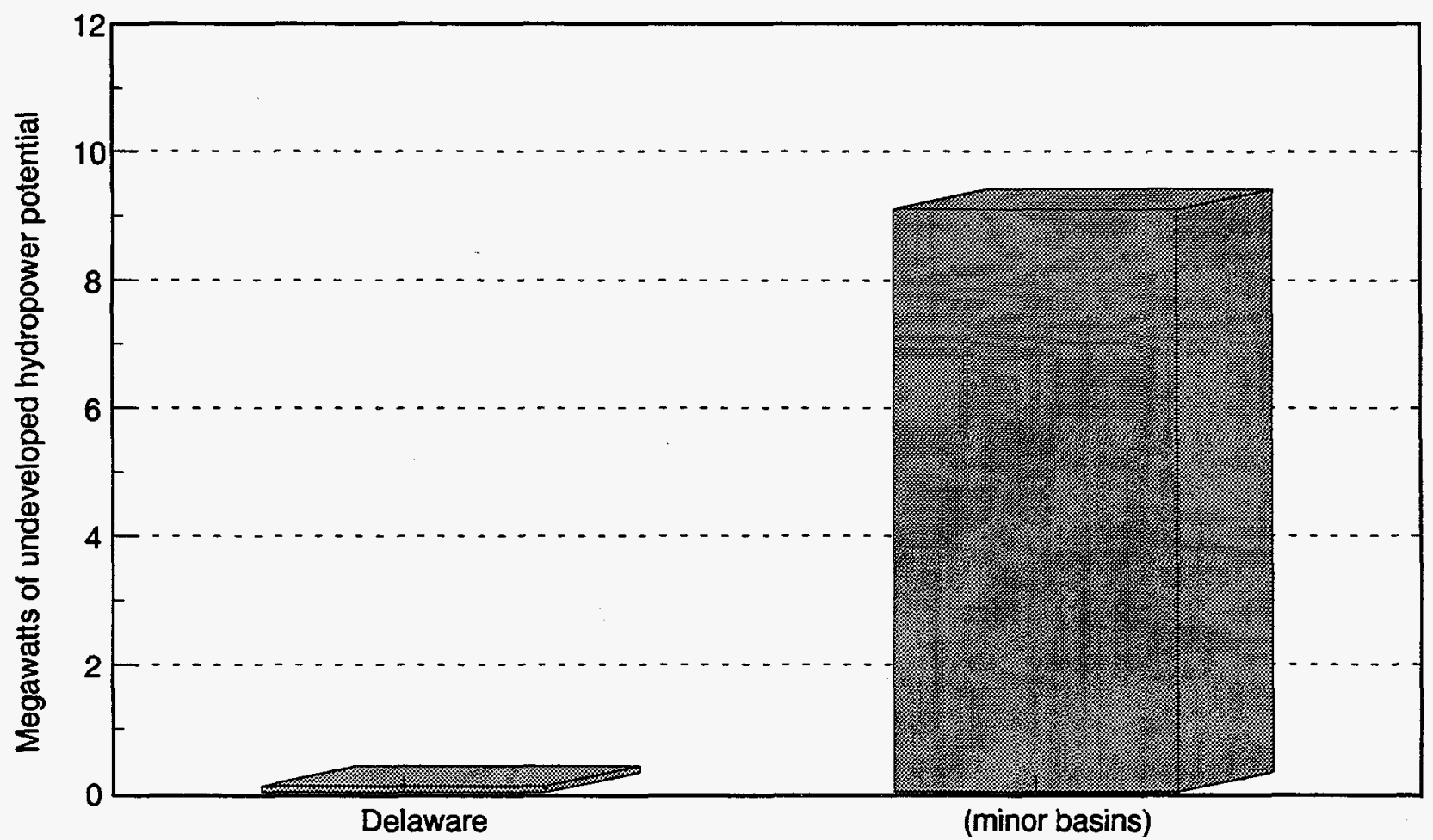

Figure 6. Megawatts of HES-modeled undeveloped hydropower potential in the New Jersey river basins. 


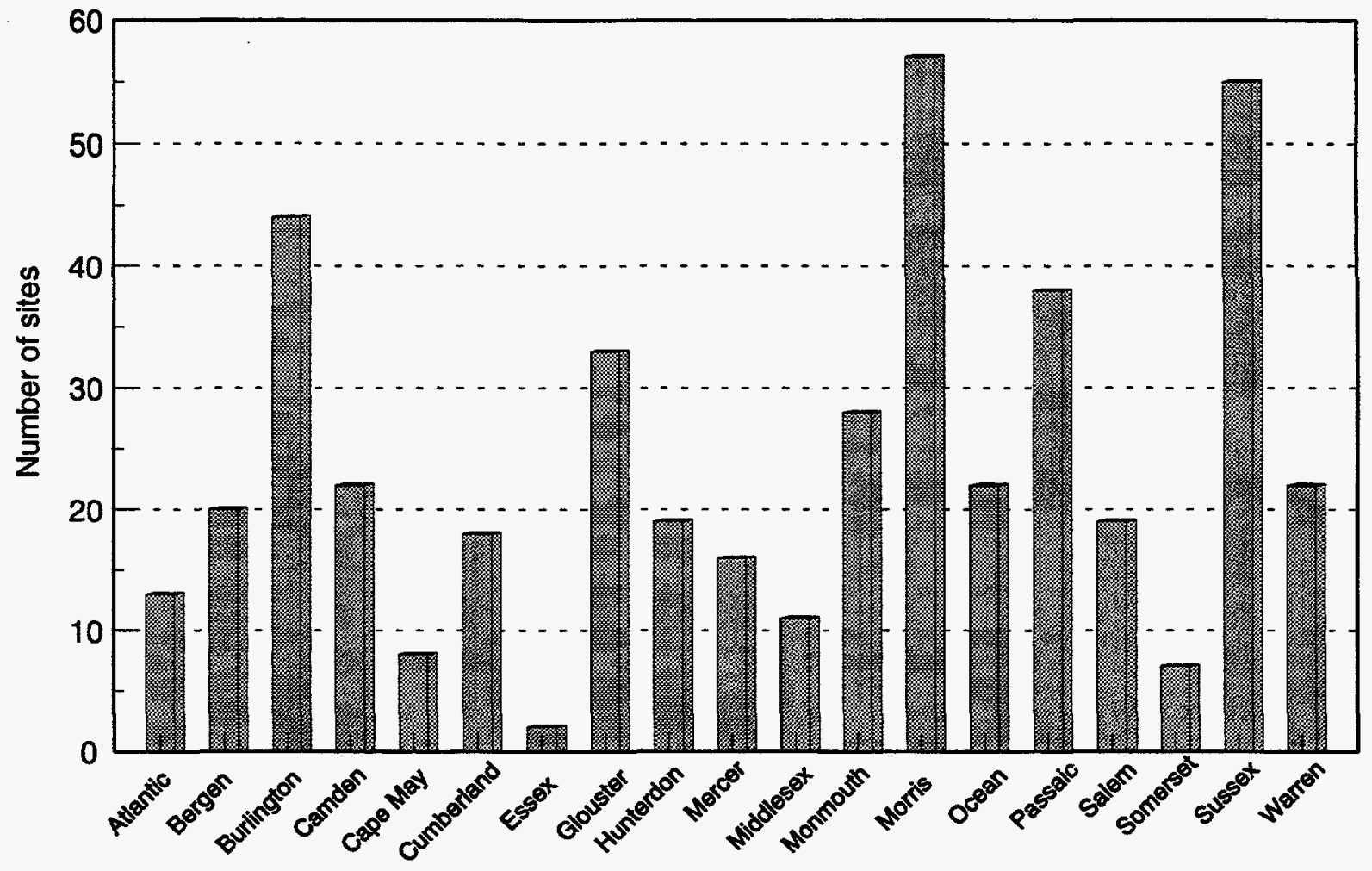

Figure 7. Number of small sites per county.

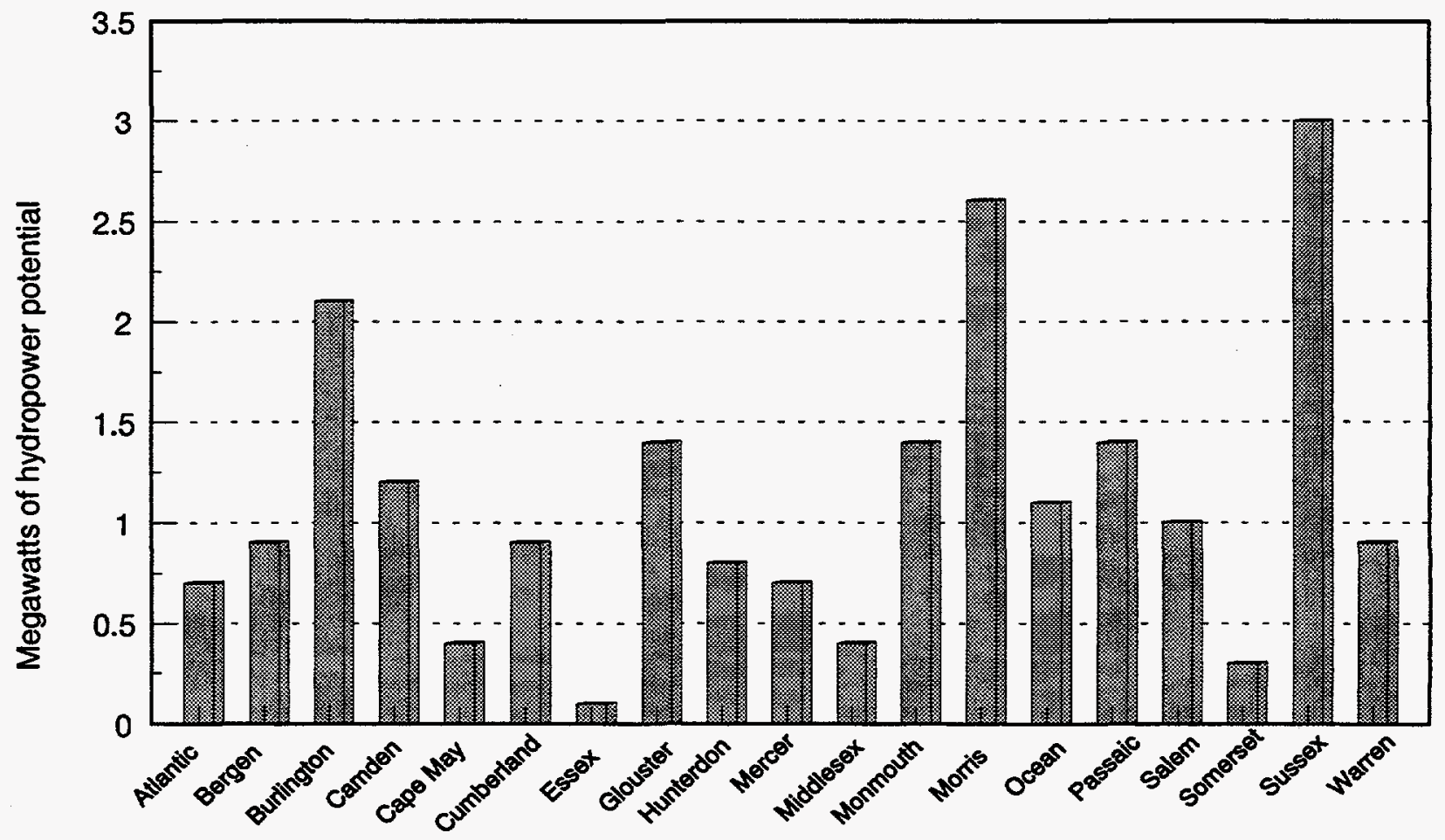

Figure 8. Nonmodeled hydropower potential capacity per county for the small sites. 
Telephone Orders-(703) 487-4650. NTIS sales desk and customer services are available between 8:30 a.m. and 5:00 p.m., Eastern Standard Time.

Fax-(703) 321-8547. Customers may fax their orders to NTIS. These orders may be charged to a NTIS deposit account, American Express, VISA, or MasterCard.

Mail Orders-Mail orders should be sent to National Technical Information Service, Document Sales, 5285 Port Royal Road, Springfield, VA 22161. Call the sales desk for prices before placing an order.

Method of Payment-Customers may pay for reports (and other NTIS products and services) by (a) credit card (American Express, Visa or MasterCard); (b) check or money order on a United States bank payable to NTIS; (c) a NTIS deposit account; or, (d) by asking to be billed (add $\$ 7.50$ per order), United States, Canada, and Mexico, only.

Handling Fee-A $\$ 3.00$ handling fee per total order applies to orders from the United States, Canada, and Mexico. Handling charges do not apply to rush order service or pick-up orders.

Postage and Shipping-Orders are shipped first class mail, or equivalent, to addresses in the United States, Canada, and Mexico.

Order Turnaround Time-Orders for technical reports generally are shipped within 2 to 8 days of receipt. For faster service, NTIS offers rush order service.

Rush Order Service-Call 1-800-533-NTIS. In Virginia, Canada, and Mexico call (703) 487-4700. For NTIS rush order service add $\$ 15.00$ per item. This guarantees that an order will be processed through NTIS within 24 hours of its receipt. These orders receive immediate, individual attention. The items ordered are delivered by first call mail. Call NTIS for information on rush order service for computer products.

For Help in Tracing an Order-Call (703) $487-4650$ and request the customer service option.

\section{ADDITIONAL HYDROPOWER EVALUATION SOFTWARE INFORMATION}

Additional information concerning the HES can be obtained by contacting Ben Rinehart or Jim Francfort at the addresses provided below. Copies of the software and the User's Manual may also be obtained from these individuals.

Ben Rinehart, Project Manager

Idaho National Engineering Laboratory

P.O. Box 1625, MS 3830

Idaho Falls, ID 83415-3830

(208) 526-1002

Jim Francfort

Idaho National Engineering Laboratory

P.O. Box 1625, MS 3875

Idaho Falls, ID 83415-3875

(208) 526-6787

Information concerning the State of New Jersey's involvement with the resource assessment or about the identified sites may be obtained by contacting:

William Crom

State of New Jersey

Energy Conservation Program

Board of Public Utilities

Two Gateway Center

Newark, NJ 07102

(201) 648-7252 


\section{REFERENCES}

Francfort, J. E., S. D. Matthews, and B. N. Rinehart, 1991, Hydropower Evaluation Software User's Manual, DOE/ID-10338, Idaho National Engineering Laboratory, Idaho Falls, Idaho.

Conner, A. M., J. E. Francfort, and B. N. Rinehart, 1996, Uniform Criteria for U.S. Hydropower Resource Assessment, Hydropower Evaluation Software Status Report-II, DOE/ID 10430.1, Idaho National Engineering Laboratory, Idaho Falls, Idaho. 

Appendix A

Summary Report

A-1 


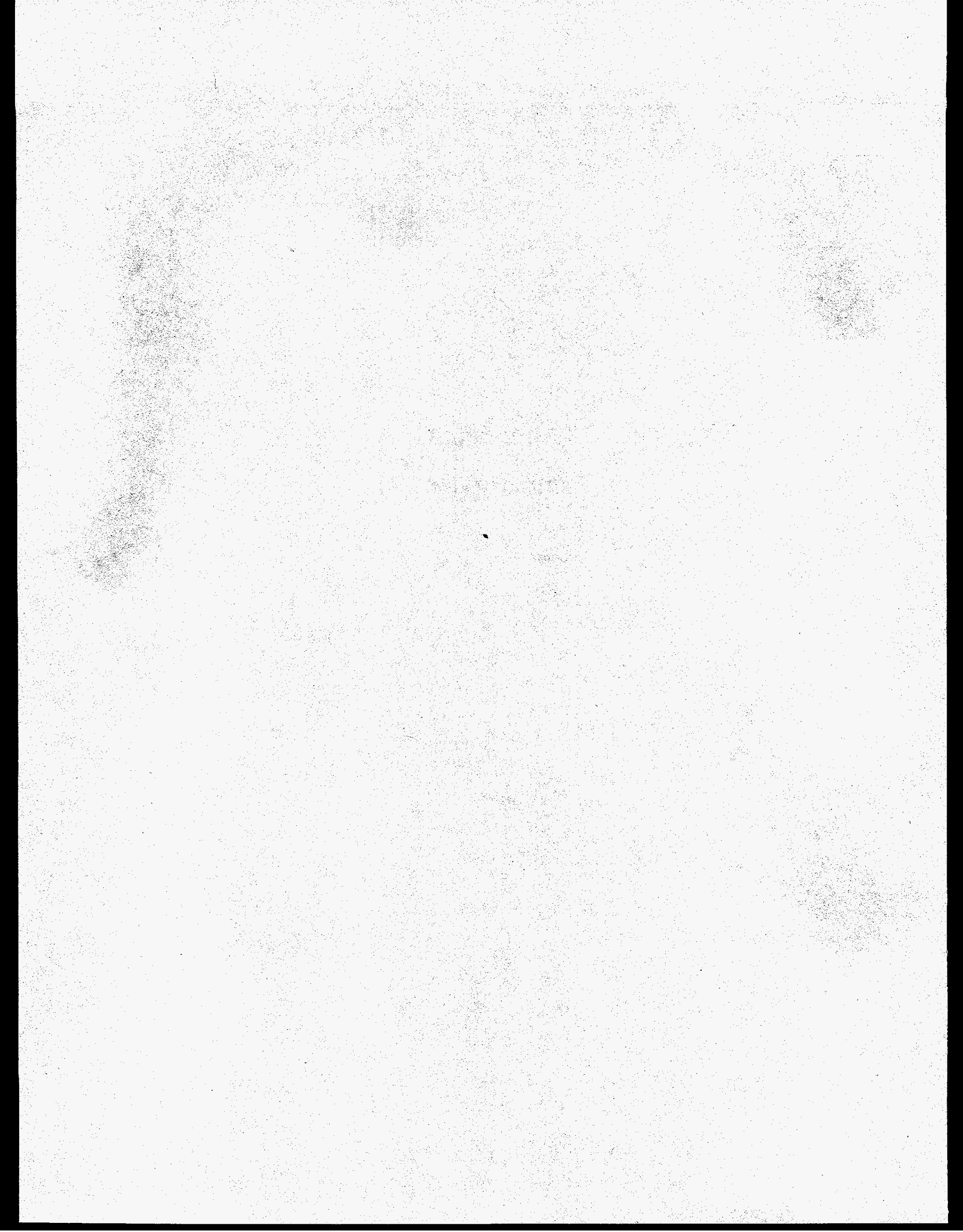




\begin{tabular}{|c|c|c|c|c|}
\hline state & $\begin{array}{l}\text { Y D R O P O } \\
\text { Category }\end{array}$ & $\begin{array}{l}\mathrm{R} \quad \mathrm{C} \mathrm{A} \\
\text { Number of } \\
\text { Projects }\end{array}$ & $\begin{array}{l}\text { T Y S U M } \\
\text { Name Plate } \\
\text { Capacity (KW) }\end{array}$ & $\begin{array}{l}02 / 15 / 96 \\
\text { R Y } \\
\text { HES Adjusted } \\
\text { Capacity (KW) }\end{array}$ \\
\hline \multirow[t]{4}{*}{ NJ } & with Power & 0 & 0 & 0 \\
\hline & w/O Power & 9 & 6145 & 5313.25 \\
\hline & Undeveloped & 3 & 4595 & 4135.50 \\
\hline & $\begin{array}{l}\text { STATE } \\
\text { TOTAL }\end{array}$ & 12 & 10740 & 9448.75 \\
\hline
\end{tabular}



Appendix B

River Basins Report

B-1 


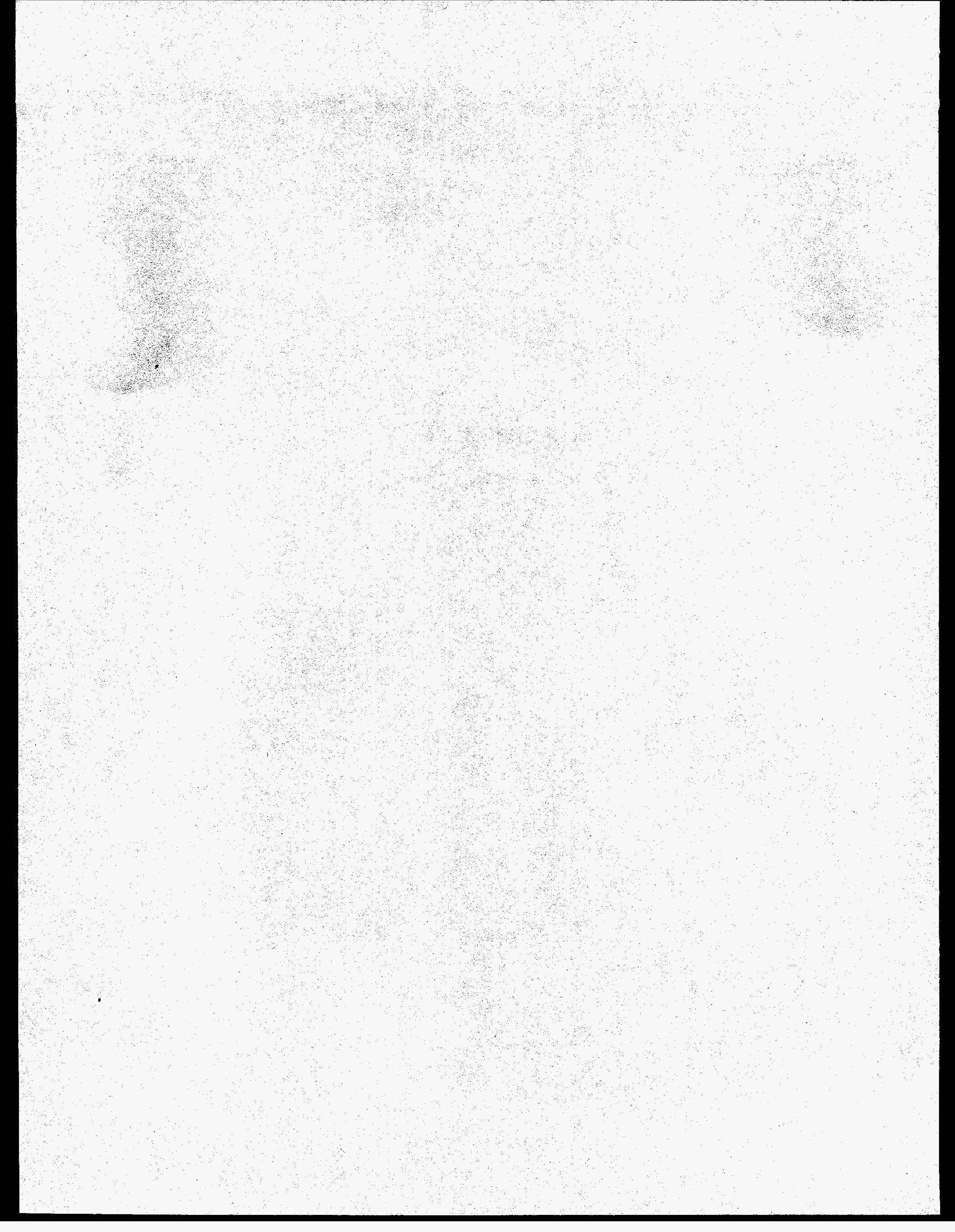




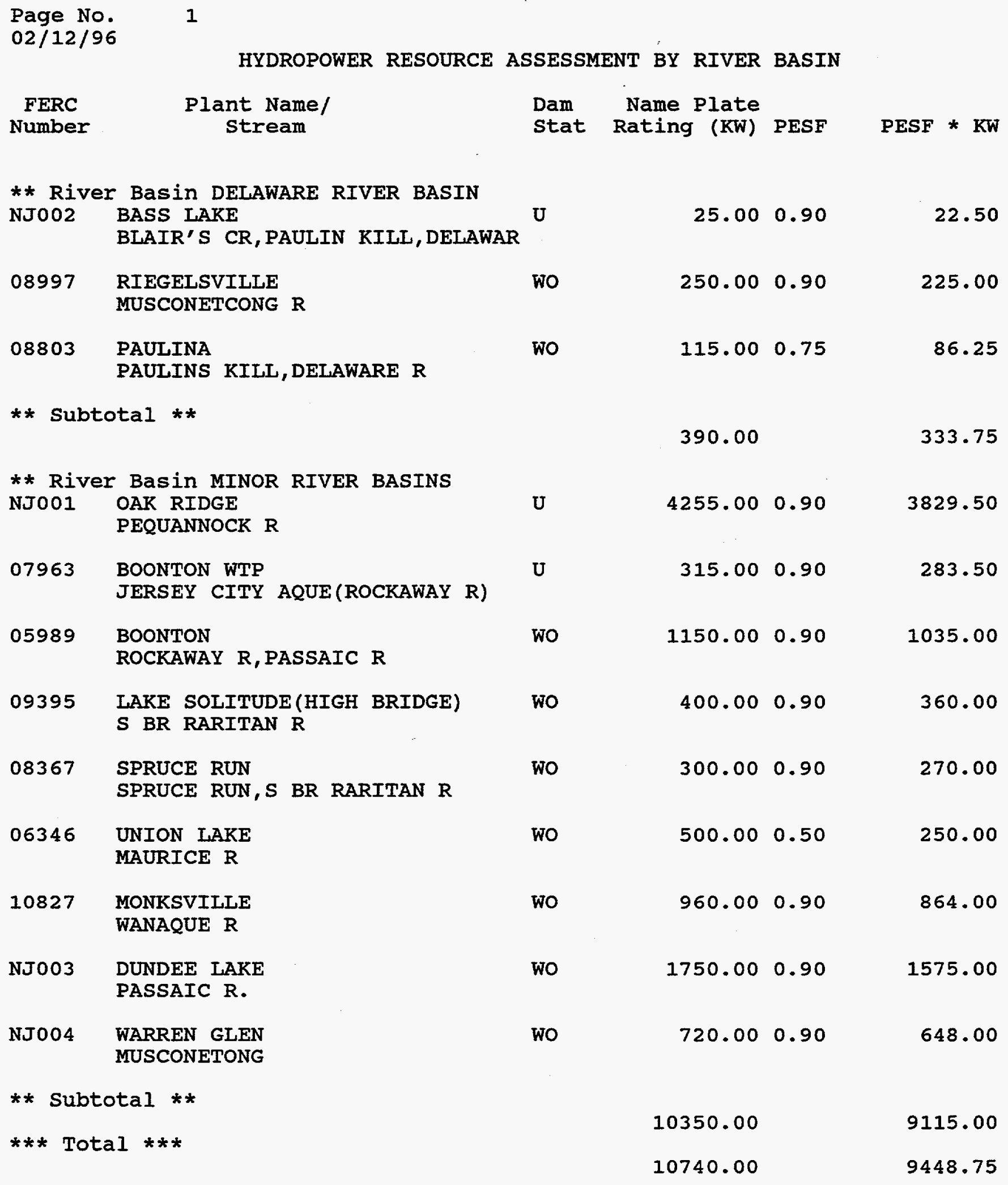



Appendix C

New Jersey Sites List

C-1 

Page No.

02/12/96
1

HYDROPOWER RESOURCE ASSESSMENT BY FERC NUMBER

Plant Name/

Stream

ST $\begin{aligned} & \text { Dam } \\ & \text { Stat }\end{aligned}$

Name Plate

Rating (KW) PESF

PESF * KW

** FERC Number 07963

BOONTON WTP

JERSEY CITY AQUE (ROCKAWAY R)

NJ U

$315.00 \quad 0.90$

283.50

** Subtotal **

315.00

283.50

** FERC Number NJ001

OAK RIDGE

PEQUANNOCK $\mathbf{R}$

NJ U

$4255.00 \quad 0.90$

3829.50

** Subtotal **

4255.00

3829.50

** FERC Number NJ002

BASS LAKE

BLAIR'S CR, PAULIN KILL, DELAWAR

NJ U

$25.00 \quad 0.90$

22.50

** Subtotal **

** FERC Number 05989

BOONTON

ROCKAWAY R,PASSAIC R

NJ wo

25.00

22.50

** Subtotal **

** FERC Number 06346

UNION LAKE

MAURICE $\mathrm{R}$

NJ wo

1150.00

1035.00

** Subtotal **

** FERC Number 08367

SPRUCE RUN

SPRUCE RUN, S BR RARITAN R

NJ พO

** Subtotal **

** FERC Number 08803

PAULINA

PAULINS KILL, DELAWARE R
NJ WO

300.00

270.00

$115.00 \quad 0.75$

86.25
250.00

250.00

270.00

$300.00 \quad 0.90$ 
HYDROPOWER RESOURCE ASSESSMENT BY FERC NUMBER

\section{Plant Name/ stream}

** Subtotal **

** FERC Number 08997

RIEGELSVILIE MUSCONETCONG $\mathbf{R}$

** Subtotal **

** FERC Number 09395

LAKE SOLITUDE (HIGH BRIDGE)

$S$ BR RARITAN R

** Subtotal **

** FERC Number 10827

MONKSVILLE

WANAQUE $R$

** Subtotal **

** FERC Number NJ003

DUNDEE LAKE

PASSAIC R.

** Subtotal **

** FERC Number NJ0O4

WARREN GLEN

MUSCONETONG

** Subtotal **

*** Total ***

\author{
Dam Name Plate \\ ST Stat Rating (KW) PESF \\ PESF * KW
}

115.00

86.25

NJ wo

$250.00 \quad 0.90$

225.00

250.00

225.00

NJ Wo

$400.00 \quad 0.90$

360.00

400.00

360.00

NJ wo

$960.00 \quad 0.90$

864.00

960.00

864.00

NJ พo

$1750.00 \quad 0.90$

1575.00

1750.00

1575.00

NJ wo

$720.00 \quad 0.90$

648.00

720.00

648.00

10740.00

9448.75 


\section{Appendix D}

Individual Resource Database List 


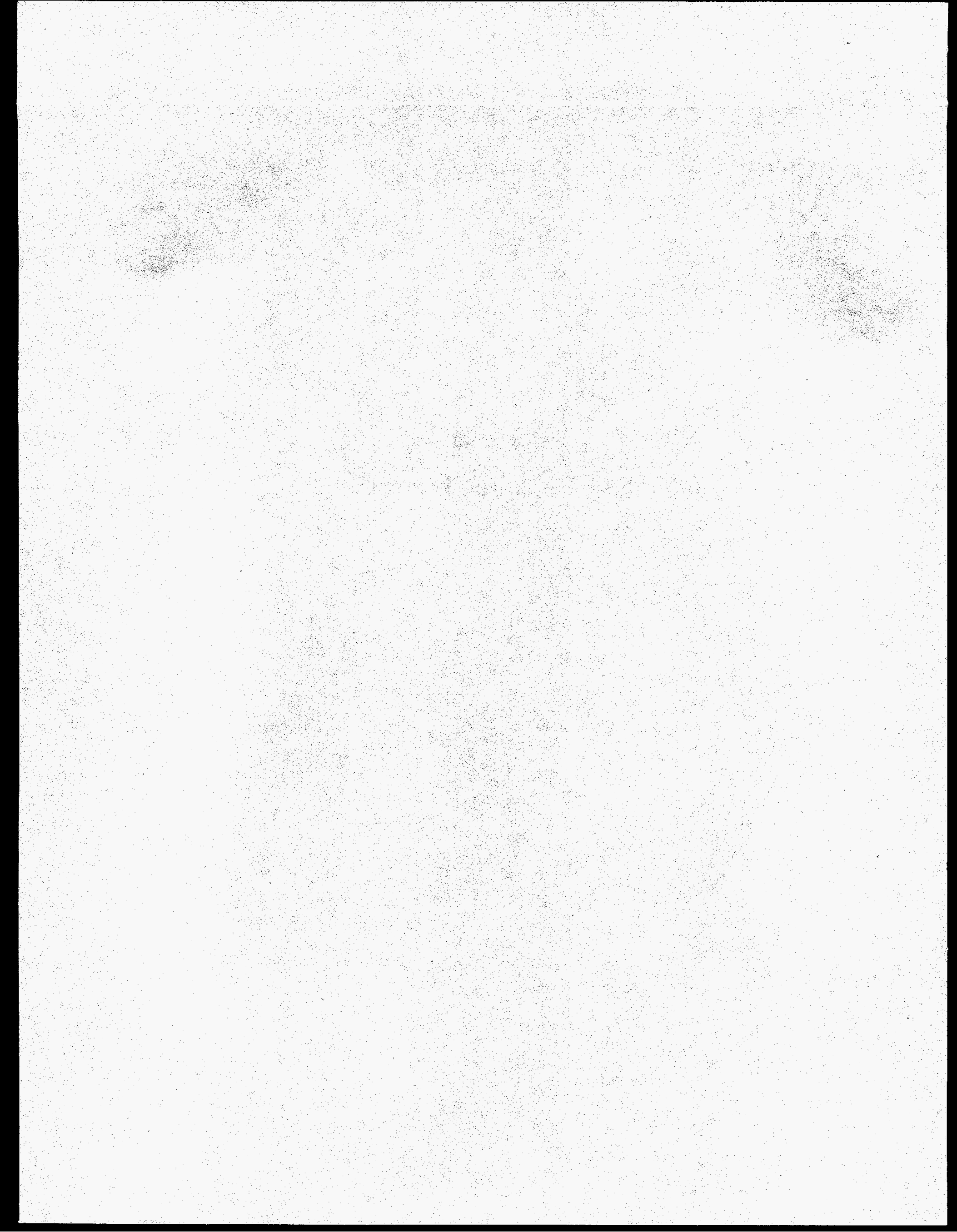


DATE: $02 / 12 / 96$

PAGE NO: 1

FERC

Number

05989

BOONTON

county Name

MORRIS
Plant Name

Class

M

JERSEY

Owner Name

M JERSEY CITY, CITY OF

Name Plate

Rating (KW)

PESF

PESF*KW

1150.00

$0.90 \quad 1035.00$
Annual Energy

Rating (MWh)

5000.00

Latitude $\quad 4056.00$

River Basin

Status Longitude 7424.00
Unit

Plant

Type

Type

C

STG

Project status

XX

Factor

wild/Scenic Protection

wild/Scenic Tributary or

Upstream/Downstream

wild/Scenic Location

Cultural Value

Fish Presence Value

Geologic Value

Historic Value

other Value

Recreation Value

Scenic value

0.90

\section{Exist Prob}

0.90

wildlife Value Threatened/Endangered Fish Threatened/Endangered wildlife

0.90 Federal Land code 103

0.90 Federal Land code 104

0.90 Federal Land code 105

0.90 Federal Land Code 106

0.90 Federal Land code 107

0.90 Federal Land code 108

0.90 Federal Land code 198
PESF Annual Energy Rating (MWh)

$$
4500
$$

State

Name

NJ

Exist Prob

0.90

0.90

0.90

0.90

0.90

0.90

0.90

0.90

0.90

0.90 
RES O UR C E D A T A B A S E I I S T I N G

\section{DATE: $02 / 12 / 96$}

FERC

Number

Plant Name

06346 UNION IAKE

county Name

CUMBERLAND

Class Owner Name

R WAWA, INC
State Name

NJ

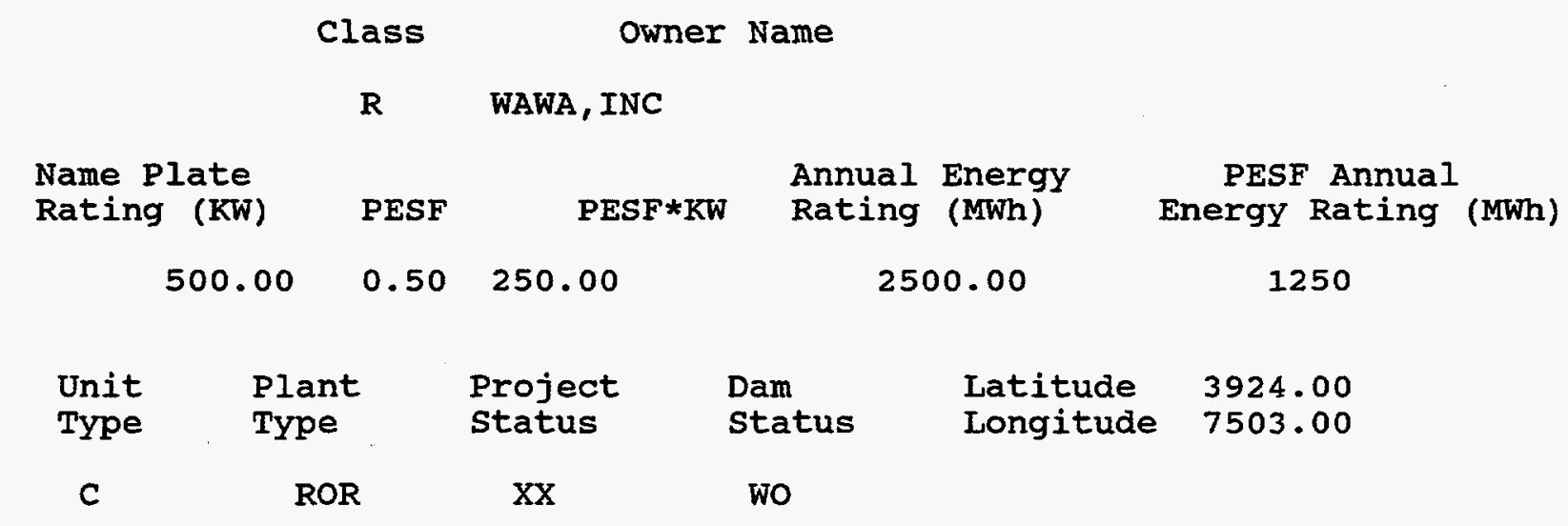

Factor

Exist Prob

Factor

Exist Prob

Wild/Scenic Protection

wild/Scenic Tributary or

0.90 Wildlife value Threatened/Endangered Fish

0.90

0.90

Upstream/Downstream Threatened/Endangered wildlife

0.90

wild/Scenic Location

$\mathrm{Y} \quad 0.75$
$\mathrm{Y} \quad 0.75$
0.90
0.90
0.90
0.90
0.90
0.90

Federal Land code 103

0.90

Federal Land Code 104

0.90

Federal Land Code 105

0.90

Fish Presence Value

Federal Land Code 106

0.90

Geologic Value

Federal Land Code 107

0.90

other Value

Recreation Value

Federal Land Code 108

0.90

Scenic Value

Federal Land Code 198

0.90 

R E S O U R C E
D A T A B A $S$ E
L I S T I N G

DATE : $02 / 12 / 96$

PAGE NO: 3

FERC

Number

Plant Name

stream

state

07963 BOONTON WTP

JERSEY CITY AQUE (ROCKAWAY R)

Name

county Name

River Basin

MORRIS

MINOR RIVER BASINS

\begin{tabular}{cc} 
Class & \multicolumn{2}{c}{ Owner Name } \\
M & JERSEY CITY, CITY OF
\end{tabular}

Name Plate $\begin{array}{ll}\text { PESF } & \text { PESF*KW } \\ \text { Rating (MWh) }\end{array}$

PESF Annual

Rating (KW)

$0.90 \quad 283.50$

1708.00

Energy Rating (MWh)

315.00

0.90283 .50

Unit Plant

Type

Type

Project

status

Dam
Status

Latitude

Longitude

1537.20

C

DIV

XX

$\mathbf{U}$

Factor

Wild/Scenic Protection

wild/Scenic Tributary or

Upstream/Downstream

wild/Scenic Location

Cultural Value

Fish Presence Value

Geologic Value

Historic Value

other Value

Recreation Value

Scenic Value
Exist Prob

0.90

Wildlife Value

Threatened/Endangered Fish

Threatened/Endangered Wildlife

0.90 Federal Land Code 103

0.90 Federal Land Code 104

0.90 Federal Land Code 105

0.90 Federal Land Code 106

0.90 Federal Land Code 107

0.90 Federal Land Code 108

0.90 Federal Land Code 198

0.90
Exist Prob

0.90

0.90

0.90

0.90

0.90

0.90

0.90

0.90

0.90

0.90 

R E S O U R C E
D A T A B A S E
L I S T I N G

DATE: $02 / 12 / 96$

PAGE NO: 4

FERC

Number

Plant Name

stream

State

08367 SPRUCE RUN

SPRUCE RUN, S BR RARITAN R

Name

county Name

River Basin

HUNTERDON

MINOR RIVER BASINS

\section{Class Owner Name}

R SPRUCE RUN HYDROPOWER ASSOC

\begin{tabular}{|c|c|c|c|c|}
\hline $\begin{array}{l}\text { Name Plate } \\
\text { Rating (KW) }\end{array}$ & PESF & PESF*KW & $\begin{array}{l}\text { Annual Energy } \\
\text { Rating (MWh) }\end{array}$ & $\begin{array}{l}\text { PESF Annual } \\
\text { Energy Rating (MWh) }\end{array}$ \\
\hline 300.00 & 0.90 & 270.00 & 1300.00 & 1170 \\
\hline
\end{tabular}

$\begin{array}{ccclll}\text { Unit } & \text { Plant } & \text { Project } & \text { Dam } & \text { Latitude } & 4039.00 \\ \text { Type } & \text { Type } & \text { Status } & \text { Status } & \text { Longitude } & 7455.00 \\ \text { C } & \text { ROR } & \text { XX } & \text { WO } & \end{array}$

Factor

Exist Prob

Factor

Exist Prob

0.90 Wildlife Value Threatened/Endangered Fish Threatened/Endangered Wildiife

0.90 Federal Land Code 103

0.90 Federal Land code 104

0.90 Federal Land Code 105

0.90 Federal Land code 106

0.90 Federal Land Code 107

0.90 Federal Land Code 108

0.90 Federal Land code 198

0.90
0.90

0.90

0.90

0.90

0.90

0.90

0.90

0.90

0.90

0.90
wild/Scenic Protection Upstream/Downstream Cultural value

Fish Presence Value

Geologic Value

Other Value

Recreation value

Scenic Value 
DATE : $02 / 12 / 96$

FERC

Number

Plant Name

08803 PAULINA

County Name

WARREN

Class

$\mathbf{R}$

GREAT BEAR HYDROPWR INC

Name Plate Rating (KW)

PESF
Annual Energy

Rating (MWh)

375.00

Latitude

Longitude

4102.00

status

$\mathrm{XX}$

Status

พo
State

Name

NJ

$\begin{array}{cccccc}\text { Unit } & \text { Plant } & \text { Project } & \text { Dam } & \text { Latitude } & 4102.00 \\ \text { Type } & \text { Type } & \text { Status } & \text { Status } & \text { Longitude } & 7448.00 \\ \text { C } & \text { ROR } & \text { XX } & \text { wo } & & \end{array}$

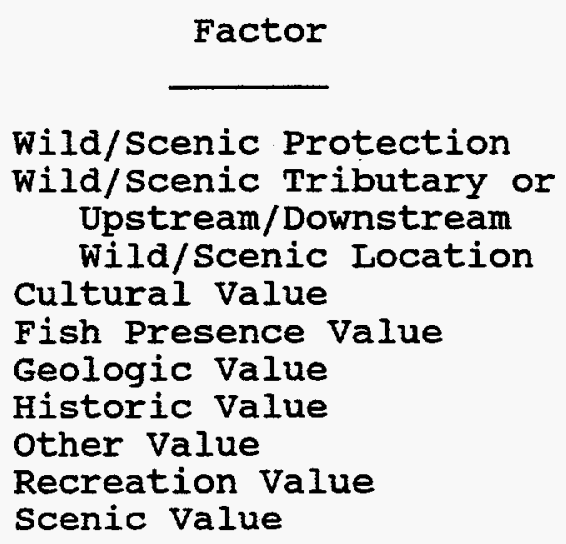

Exist Prob

Factor

Exist Prob

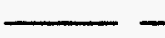

\subsection{0}

$\mathrm{Y}$

0.75

0.90

0.90

0.90

0.90

0.90

0.90

0.90

Wildife Value

Threatened/Endangered Fish

Threatened/Endangered Wildlife

Federal Land Code 103

Federal Land Code 104

Federal Land Code 105

Federal Land Code 106

Federal Land Code 107

Federal Land Code 108

Federal Land Code 198
PESF Annual Energy Rating (MWh)

281.25 

R E S O U R C E
D A T A B A S E
I I S T I N G

DATE: $02 / 12 / 96$

PAGE NO: 6

FERC

Number

08997
Plant Name

RIEGELSVILLE

county Name

WARREN
State

stream

MUSCONETCONG $\mathbf{R}$

NJ

$$
\begin{array}{cc}
\text { Class } & \text { Owner Name } \\
\text { R } & \text { GREAT BEAR HYDROPWR INC }
\end{array}
$$

$\begin{array}{lll}\text { Name Plate } & & \text { Annual Energy } \\ \text { Rating (KW) } & \text { PESF } & \text { PESF*KW Rating (MWh) }\end{array}$

250.00
450.00

\section{Latitude}

Longitude

4038.00

Status

WO
PESF Annual Energy Rating (MWh)

\begin{tabular}{|c|c|c|c|c|c|}
\hline $\begin{array}{l}\text { Unit } \\
\text { Type }\end{array}$ & $\begin{array}{l}\text { Plant } \\
\text { Type }\end{array}$ & $\begin{array}{l}\text { Project } \\
\text { status }\end{array}$ & $\begin{array}{l}\text { Dam } \\
\text { status }\end{array}$ & $\begin{array}{l}\text { Latitude } \\
\text { Longitude }\end{array}$ & $\begin{array}{l}4038.00 \\
7511.00\end{array}$ \\
\hline c & ROR & $\mathrm{XX}$ & wo & & \\
\hline
\end{tabular}

405

Factor

Exist Prob

Factor

Exist Prob

0.90 Wildlife Value Threatened/Endangered Fish Threatened/Endangered Wildlife

0.90 Federal Land code 103

0.90 Federal Iand Code 104

0.90 Federal Land Code 105

0.90 Federal Land code 106

0.90 Federal Land code 107

0.90 Federal Land Code 108

0.90 Federal Land Code 198
0.90

0.90

0.90

0.90

0.90

0.90

0.90

0.90

0.90

0.90

Recreation Value

Scenic Value

0.90 
DATE : $02 / 12 / 96$

PAGE NO: 7

FERC

Number

09395

Plant Name

stream

State

Name

LAKE SOLITUDE(HIGH BRIDGE) S BR RARITAN R

NJ

\author{
River Basin
}

HUNTERDON

MINOR RIVER BASINS

Class Owner Name

R SMITCHIE HYDROELEC CO INC

Name Plate Rating ( $\mathrm{KW}$ )

PESF

Annual Energy

400.00

$0.90 \quad 360.00$

2500.00

PESF Annual Energy Rating (MWh)

$\begin{array}{cccll}\text { Unit } & \text { Plant } & \text { Project } & \text { Dam } & \text { Latitude } \\ \text { Type } & \begin{array}{l}\text { Type } \\ \text { Status }\end{array} & \text { Status } & \text { Longitude } & 7455.00 \\ \text { C } & \text { STG } & \text { XX } & \text { wo } & \end{array}$

\section{Factor}

wild/Scenic Protection

wild/Scenic Tributary or Upstream/Downstream wild/Scenic Location

cultural value

Fish Presence Value

Geologic Value

Historic Value

other Value

Recreation Value

Scenic value
Exist Prob

0.90

Wildife value

Threatened/Endangered Fish

Threatened/Endangered Wildlife

0.90 Federal Land code 103

0.90 Federal Land Code 104

0.90 Federal Land code 105

0.90 Federal Land code 106

0.90 Federal Land code 107

0.90 Federal Land code 108

0.90 Federal Iand code 198
Exist Prob

0.90

0.90

0.90

0.90

0.90

0.90

0.90

0.90

0.90

0.90 
R E S O UR C E D A T A B A E L I S T I N G

DATE: $02 / 12 / 96$

PAGE NO: 8

FERC

Number

10827
Plant Name

MONKSVIILE

County Name

PASSAIC state

Name

NJ

WANAQUE $\mathrm{R}$

stream

River Basin

MINOR RIVER BASINS

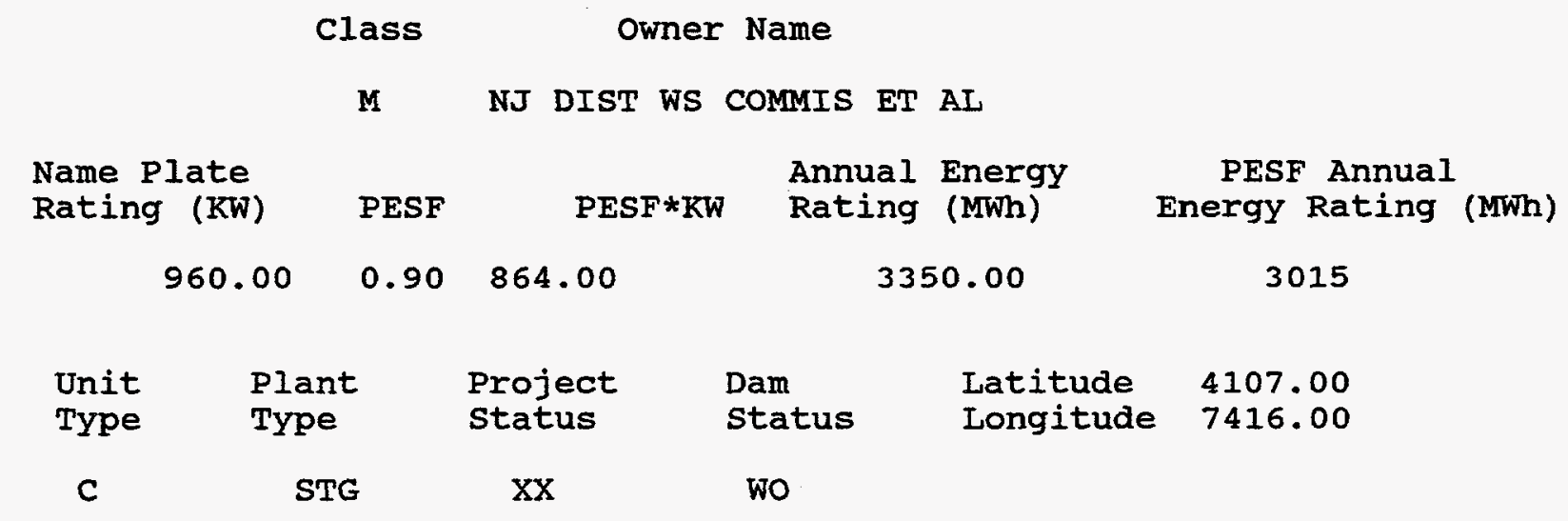

Factor

Wild/Scenic Protection

wild/Scenic Tributary or Upstream/Downstream

wild/Scenic Location Cultural value

Fish Presence Value

Geologic Value

Historic Value

other Value

Recreation Value

Scenic Value
Exist Prob

0.90

Wildife value

Threatened/Endangered Fish

Threatened/Endangered Wildlife

0.90 Federal Land Code 103

0.90 Federal Land Code 104

0.90 Federal Land Code 105

0.90 Federal Land Code 106

0.90 Federal Land Code 107

0.90 Federal Land Code 108

0.90 Federal Land Code 198

0.90
Exist Prob

0.90

0.90

0.90

0.90

0.90

0.90

0.90

0.90

0.90

0.90 
DATE : $02 / 12 / 96$

FERC

Number

Plant Name

NJ001 OAK RIDGE

County Name

MORRIS

Class

Owner Name

M NEWARK MUNICIPAL UTILITY

Name Plate

Rating ( $\mathrm{KW})$

4255.00

PESF

PESF*KW

Annual Energy

Rating (MWh)

10400.00

$0.90 \quad 3829.50$

STG

\section{Factor}

Wild/Scenic Protection

Wild/Scenic Tributary or Upstream/Downstream wild/Scenic Location

Cultural value

Fish Presence Value

Geologic Value

Historic Value

other Value

Recreation value

Scenic Value
River Basin

MINOR RIVER BASINS
$\mathbf{U}$

$\begin{array}{llllll}\text { Unit } & \text { Plant } & \text { Project } & \text { Dam } & \text { Latitude } & 4102.00 \\ \text { Type } & \text { Type } & \text { Status } & \text { Status } & \text { Longitude } & 7430.00\end{array}$

$\begin{array}{llllll}\text { Unit } & \text { Plant } & \text { Project } & \text { Dam } & \text { Latitude } & 4102.00 \\ \text { Type } & \text { Type } & \text { Status } & \text { Status } & \text { Longitude } & 7430.00\end{array}$

$\begin{array}{llllll}\text { Unit } & \text { Plant } & \text { Project } & \text { Dam } & \text { Latitude } & 4102.00 \\ \text { Type } & \text { Type } & \text { Status } & \text { Status } & \text { Longitude } & 7430.00\end{array}$

$\begin{array}{llllll}\text { Unit } & \text { Plant } & \text { Project } & \text { Dam } & \text { Latitude } & 4102.00 \\ \text { Type } & \text { Type } & \text { Status } & \text { Status } & \text { Longitude } & 7430.00\end{array}$

PAGE NO: 9

state

Name

NJ
PESF Annual Energy Rating (MWh)

9360
Exist Prob

0.90

Wildife Value Threatened/Endangered Fish Threatened/Endangered Wildife

0.90 Federal Land Code 103

0.90 Federal Land Code 104

0.90 Federal Land Code 105

0.90 Federal Land Code 106

0.90 Federal Land Code 107

0.90 Federal Land Code 108

0.90 Federal Land Code 198
Exist Prob

0.90

0.90

0.90

0.90

0.90

0.90

0.90

0.90

0.90

0.90 
RESOU R C E D A T A B A S E I I S T I N G

DATE: $02 / 12 / 96$

PAGE NO: 10

FERC

Number

Plant Name

Stream

State

NJ002 BASS I.AKE

BLAIR'S CR, PAULIN KILL,DELAWAR

Name

county Name

River Basin

WARREN

DELAWARE RIVER BASIN

Class owner Name

R PRINCETON BLAIRSTOWN CCENTER

Name Plate

$\begin{array}{lll} & \text { Annual Energy } \\ \text { PESF } & \text { PESF*KW } & \text { Rating (MWh) }\end{array}$

PESF Annual

Rating (KW)

$0.90 \quad 22.50$

1100.00

990

$\begin{array}{cc}\text { Unit } & \begin{array}{l}\text { Plant } \\ \text { Type }\end{array} \\ \text { Type } \\ \text { C } & \text { ROR }\end{array}$

$\begin{array}{lll}\text { Dam } & \text { Latitude } & 4102.00 \\ \text { Status } & \text { Longitude } & 7457.00\end{array}$

Factor

Exist Prob

Factor

Exist Prob

Wild/Scenic Protection

wild/Scenic Tributary or

Upstream/Downstream

wild/Scenic Location

Project

status

U

Cultural Value

Fish Presence Value

Geologic Value

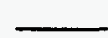

0.90

Wildlife Value

Threatened/Endangered Fish

0.90

Threatened/Endangered Wildlife

0.90

0.90 Federal Land Code 103

0.90

0.90 Federal Land code 104

0.90

0.90

Federal Land Code 105

0.90

0.90 Federal Land code 106

0.90 Federal Land Code 107

0.90

Historic Value

0.90

Federal Land Code 108

0.90

other Value

Recreation Value

0.90

Federal Land code 198

0.90

0.90

Scenic Value

0.90

0.90 
DATE: $02 / 12 / 96$

FERC

Number

Plant Name

NJ003 DUNDEE LAKE

county Name

BERGEN
PAGE NO: 11

state

Name

PASSAIC $\mathbf{R}$.

NJ
River Basin

MINOR RIVER BASINS

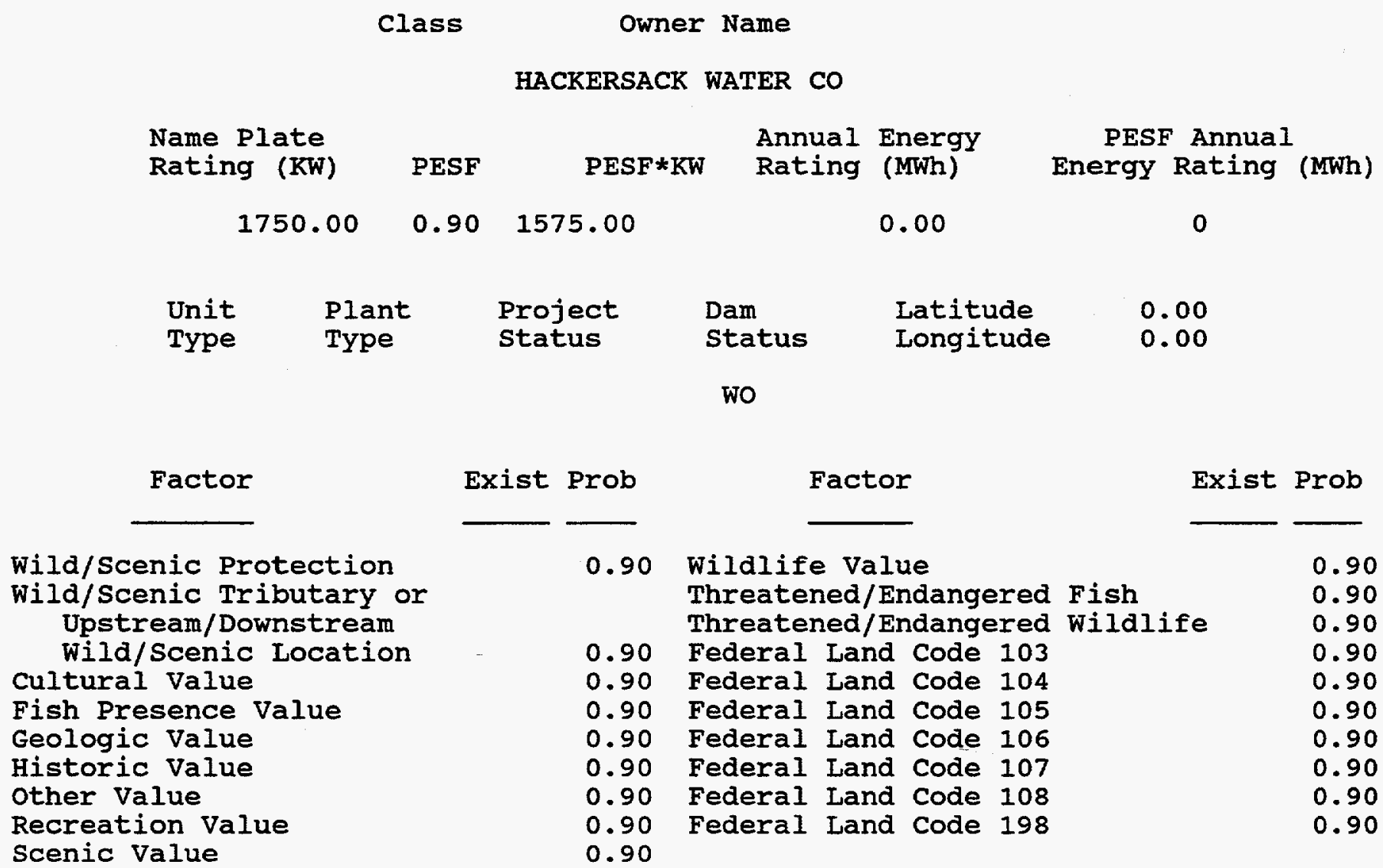



R E S O U R C E
D A T A B A S E
I I S T I N G

DATE: $02 / 12 / 96$

PAGE NO: 12

FERC

Number

Plant Name

stream

State

NJ004 WARREN GLEN

MUSCONETONG

Name

NJ

county Name

River Basin

WARREN

MINOR RIVER BASINS

Class Owner Name

RIEGEI PROGDUCTS CORP

Name Plate

Rating (KW)

PESF

Annual Energy

PESF* KW

Rating (MWh)

PESF Annual

720.00

$0.90 \quad 648.00$

0.00

0

$\begin{array}{lll}\text { Unit } & \text { Plant } & \text { Project } \\ \text { Type } & \text { Type } & \text { status }\end{array}$

$\begin{array}{ll}\text { Dam } & \text { Latitude } \\ \text { Status } & \text { Longitude }\end{array}$

0.00

WO

Factor

wild/Scenic Protection

wild/Scenic Tributary or Upstream/Downstream

wild/scenic Location

Cultural value

Fish Presence Value

Geologic Value

Historic Value

other value

Recreation Value

Scenic Value
Exist Prob

0.90

Wildlife Value Threatened/Endangered Fish Threatened/Endangered Wildlife

0.90 Federal Land code 103

0.90 Federal Land code 104

0.90 Federal Land Code 105

0.90 Federal Land Code 106

0.90 Federal Land Code 107

0.90 Federal Land code 108

0.90 Federal Land code 198

0.90
Exist Prob

0.90

0.90

0.90

0.90

0.90

0.90

0.90

0.90

0.90

0.90 


\section{Appendix E}

\section{List of $\mathbf{4 6 9}$ Small Sites}

E-1 


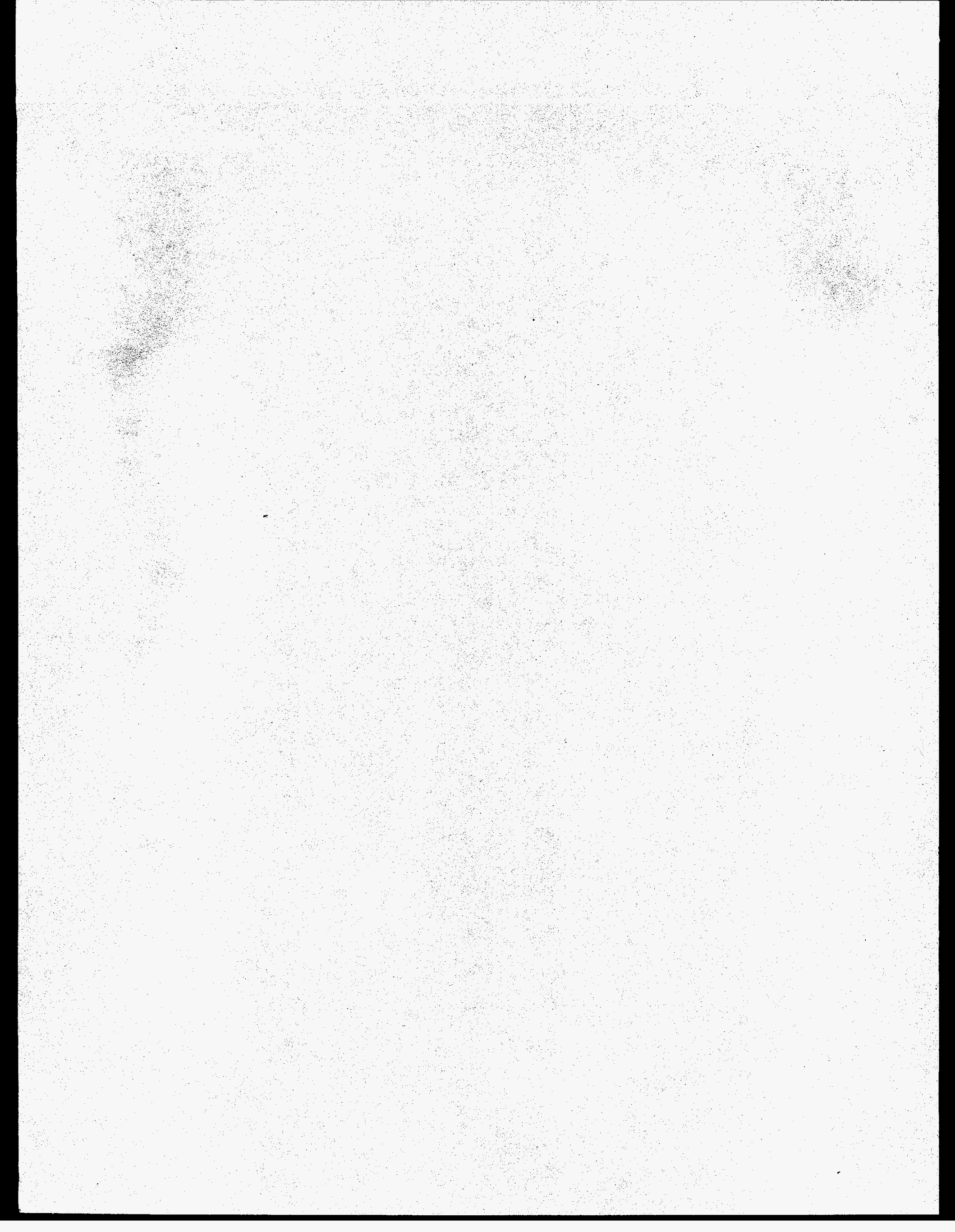




\begin{tabular}{|c|c|c|c|c|c|c|}
\hline damname & kw & ght & river & county & nearbytown & ownername \\
\hline BSALON DOUGHTY POND DAM & 29.2 & 16 & ABSECON CREEK & ATLANTIC & ABSECON & ATLANTIC CITY ENERGY OFFICE \\
\hline DOUGHTY POND UPPER DAM & 12.4 & 13 & ABSECON SO. BRANCH & ATLANTIC & ABSECON & ATLANTIC CITY \\
\hline LILY LAKE DAM & 70.0 & 7 & DOUGHTY CREEK & ATLANTIC & GALLOWAY & GALLOWAY TUP. \\
\hline ENGLISH CREEK POND DAM & 55.0 & 10 & ENGLISH CREEK & ATLANTIC & ENGLISH CREEK & MRS. CLIFFORD HOGAN \\
\hline CEDAR LAKE DAM & 80.0 & 8 & GREAT EGG HARBOR R. TRIBUTARY & ATLANTIC & BUENA VISTA & LAMREHCE TWP. CEDARVILLEE \\
\hline LAKE GEORGE DAM & 13.5 & 8 & GREAT EGG HARBOR RIVER & ATLANTIC & PENHY POT & COLINGS LAKES DEVEL. CO. \\
\hline UNKOWN & 90.0 & 6 & GREAT SWAMP BRANCH & ATLANTIC & PLEASANT MILLS & BILL THOMS \\
\hline HAMMONTON LAKE DAM & 2.7 & 9 & HAMMONTOH CR. & ATLANTIC & ELWOOD & STATE OF NJ \\
\hline CUSHMAN LAKE DAM & 39.8 & 13 & HOSPITALITY BR. & ATLANTIC & PENNY LOT & COLLINS LAKE DEVEL. \\
\hline CUSHMAN LAKE DAM & 83.5 & 14 & HOSPITALITY BROOK & ATLANTIC & PENNY POT & COLLINGS LAKES CIVIC ASSOC \\
\hline MOSS MILL LAKE DAM & 70.0 & 7 & MORSES MILL STREAN & ATLANTIC & PT. REPUBLIC & MOSS MILL LAKE CAMPGROUND \\
\hline LAKE ALBERT DAM & 12.0 & 7 & TR. GREAT EGG HARBOR & ATLANTIC & PENAY POT & COLLINGS LAKES DEVEL. CO. \\
\hline UNKNOWN & 90.0 & 7 & TR. WATERING RACE & ATLANTIC & MAYS LANDING & R. RICHETOLA \\
\hline CAINES MILL POND DAM & 90.0 & 8 & WHITE OAK BRANCH & ATLANTIC & Folsom & \\
\hline DOUGHTY POND UPPER DAM & 12.4 & 13 & ABSECON SO. BRANCH & BERGEN & ABSECON & ATLANTIC CITY \\
\hline DARLINGTON LAKE & 77.0 & 19 & DARLINGTON LAKE & BERGEN & MAHHAH TWP. & BERGEN CITY PARK COMM. \\
\hline LAKE VREELAND DAM & 36.0 & 20 & FOX BROOK & BERGEN & CAMP GLEN GRAY & ESSEX CTY BSA \\
\hline LAKE VREELAND DAM & 36.0 & 20 & FOX BROOK & BERGEN & CAMP GLEN GRAY & ESSEX CITY BSA \\
\hline RAMBAUT LAKE & 60.0 & 11 & GOFFLE BROOK & BERGEN & WYCKOFF & ALBERT ZECHER \\
\hline MAPLE LAKE DAM & 34.0 & 16 & GOFFLE BROOK & BERGEN & MIDLAND PARK & CANTERBURY DEVELOP CORP. \\
\hline HAMMONTON LAKE DAM & 2.7 & 9 & HAMMONTON CR. & BERGEN & ELWOOD & STATE OF NJ \\
\hline WHITES POND DAM & 21.2 & 13 & HOHOKUS BR. & BERGEN & WALDWICK & BORO OF WALOWICK \\
\hline WHITES POND DAM & 57.0 & 21 & HOHOKUS BROOK & BERGEN & WALDHICK & BOROUGH OF WALOWICK \\
\hline SHADOW LAKE DAM & 55.0 & 10 & HOHOKUS CREEK & BERGEN & ALLLENDAL.E & SHADOW LAKE INC. \\
\hline CUSHMAN LAKE DAM & 39.8 & 13 & HOSPITALITY BR. & BERGEN & PENNY POT & COLLINGS LAKES DEVEL. \\
\hline ELECTRIC LIGHT POND DAM & 23.6 & 14 & PASCACK BR. & BERGEN & HILLSDALE & BORO OF PARK RIDGE \\
\hline
\end{tabular}




\begin{tabular}{|c|c|c|c|c|c|c|}
\hline WOODCLIFF LAKE DAM & 70.4 & 33 & PASCACK BR. & BERGEN & HILLSDALE & HACKENSACK MATER CO. \\
\hline SWARTSWOOD LAKE DAM & 30.2 & 16 & PAULINS KILL BR. & BERGEN & MIDDLEVILLE & NJDEP \\
\hline CRYSTAL LAKE DAM & 9.2 & 13 & RAMAPO R. & BERGEN & OAKLAND & RAMAPO MT. LAKE COUNTRY CLUB \\
\hline NO NAME & 45.0 & 6 & RAMAPO RIVER & BERGEN & OAKLAND & FRED WEHREN \\
\hline NO NAME & 45.0 & 6 & RAMAPO RIVER & BERGEN & OAKLAND & FRED WEHREN \\
\hline CRYSTAL SPRING LAKE DAM & 65.0 & 12 & RAMSEY BROOK PASSAIC R. BASIN & BERGEN & ALLENDALE & RAMSEY GOLF COUNTRY CLUB \\
\hline LAKE TAMARACK DAM & 98.0 & 11 & TR. RAMAPO RIVER & BERGEN & OAKLAND & LAKE TAMARACK ASSOC. \\
\hline LAKE TAMARACK DAM & 98.0 & 11 & TR. RAMAPO RIVER & BERGEN & OAKLAND & LAKE TAMARACK ASSOC. \\
\hline TOMLINSONS MILL DAM & 24.0 & 11 & BARTON RUN & BURLINGTON & MEDFORD & HILLIAN EVAN \\
\hline BATSTO DAM & 93.0 & 12 & BATSTO RIVER & BURLINGTON & BATSTO & STATE OF NJ \\
\hline NJ NO NAME & 70.0 & 15 & BLACK CREEK & BURLINGTON & CHESTERF IELD & \\
\hline TIMBER LAKE DAM & 11.2 & 11 & BR. OF HAYMES CREEK & BURLINGTON & PINE LAKE & BIRCHWOOD LAKES COLONY \\
\hline BALLINGER LAKE DAM & 46.0 & 14 & BRANCH HAYNES CREEK & BURLIMGTON & BIRCHWOOD LAKES & MEDFORD LAKES \\
\hline NJ NO NAME \#17 & 84.0 & 10 & BURRS MILL BROOK & BURLINGTON & VINCENTOWN & STATE OF NJ \\
\hline NO NAME DAM & 80.0 & 6 & COOPER BRANCH & BURLINGTON & UPPER MILL & STATE OF Nd \\
\hline OLD EAGLE MILL RUN & 10.0 & 10 & CROSSWOCKS CREEK & BURLINGTON & GROVEVILLE & FRANK HALPER \\
\hline HANOVER LAKE DAM & 19.6 & 10 & HARTSHORNE MLLL STREAM & BURLINGTON & BROWNS MILLS & US ARMY \\
\hline AETNA LAKE DAM & 70.4 & 10 & HAYES CREEK & BURLINGTON & MEDFORD LAKES & MEDFORD LAKES COLONY CLUB \\
\hline UPPER STOKES DAM & 50.0 & 11 & HAYNES CREEK & BURLINGTON & MEDFORD LAKES & WILLIAM STOKES \\
\hline LAKE STOCKWELL DAM & 61.0 & 12 & HAYNES CREEK & BURLINGTON & MEDFORD LAKES & YMCA CAMP \\
\hline OLIPHANTS MILL LAKE DAM & 32.2 & 11 & HAYNES CREEK & BURLINGTON & MEDFORD & OAKWOOD LAKES COUNTRY CLUB \\
\hline HOOTENS CREEK DAM & 13.0 & 9 & HOOTENS CREEK & BURLINGTON & MOORESTOWN & TWP OF MOORESTOWN \\
\hline INDIAN MILLS SAHMILL POND & 20.0 & 8 & INDIAN BROOK & BURL INGTON & INDIAN MILLS & TOWNSHIP OF SHAMONG \\
\hline PINE MILL LAKE DAM & 95.0 & 18 & JACKS RUN & BURLINGTON & NEW LISBON & BURLINGTON CITY \\
\hline BREAKNECK DAM & 15.7 & 11 & KETTLE RUN & BURLINGTON & LAKE PINE & TAUNTON LAKES CO. \\
\hline CENTENNIAL LAKE DAM & 10.4 & 13 & KETTLE RUN & BURLINGTON & TAUNTON LAKE & CENTENNIAL LAKES CO. \\
\hline PACHOANGO DAM & 86.0 & 12 & KETTLE RUN & BURLINGTON & TAUNTON LAKE & PACHOANGO CORP. \\
\hline
\end{tabular}




\begin{tabular}{|c|c|c|c|c|c|}
\hline 9.0 & 16 & KETTLE RUN & BURLINGTON & TAUNTON LAKE & BRADDOCKS MILL \\
\hline 4.0 & 10 & KETTLE RUN & BURLINGTON & TAUNTON LAKE & MARLTON LAKES CIVIL ASSOC. \\
\hline 9.0 & 18 & MILL STREAM & BURLINGTON & BURLINGTON & CITY OF BURLINGTON \\
\hline 0.0 & 25 & MILL STREAM & BURLINGTON & BURLINGTON & BURLINGTON TWP. \\
\hline 0.0 & 8 & MT. MISERY BROOK & BURLINGTON & NEW LISBON & \\
\hline 17.8 & 7 & MULLICA RIVER & BURLINGTON & PLEASANT MILLS & STATE OF NJ \\
\hline 8.0 & 13 & NEWBOLD RUN & BURLINGTON & WRIGHTSTOWN & US ARMY \\
\hline 0.0 & 8 & NO. BR. RANCOCAS CR. & BURLINGTON & MT. HOLLY & CITY OF BURLINGTON \\
\hline 1.0 & 8 & NORTH BRANCH PENNSAUKEN RIVER & BURLINGTON & MOORESTOWN & TWP OF MOORESTOWN \\
\hline 5.3 & 11 & OSWEGO RIVER & BURL INGTON & WADING RIVER & STATE OF NJ \\
\hline 0.8 & 10 & OSWEGO RIVER & BURLINGTON & MARTHA & STATE OF NJ \\
\hline 1.9 & 12 & POLE BRIDGE BR. & BURLINGTON & NEH LISBON & COUNTY LAKES INC. \\
\hline 0.4 & 12 & POLE BRIDGE BR. & BURLINGTON & NEW LISBON & FRIENDSHIP LAKES, INC. \\
\hline 5.4 & 14 & POLE BRIDGE BRANCH & BURLINGTON & NEW LISBON & COUNTRY LAKES INC. \\
\hline 2.7 & 18 & RANCOCAS CREEK & BURLINGTON & BROWNS MILLS & PEMBERTON THP. \\
\hline 4.0 & 15 & SE BRANCH HAYNES CREEK & BURLINGTON & PINES LAKE & BIRCHWOOO LAKE COUNTRY CLUB \\
\hline 0.0 & 15 & TO BLACK CREEK & BURLINGTON & CHESTERFIELD & WALTER GUZILOWSKI \\
\hline 5.0 & 8 & TO SOUTH RUN & BURL INGTON & MCGUIRE AFB & DOD USA \\
\hline 0.0 & 15 & TO SOUTH RUN & BURL INGTON & MCGUIRE AFB & DOD USA \\
\hline 1.1 & 6 & TOMHY'S BROOK & BURLINGTON & NEW GRETNA & DEPT. OF CONS. \& DEVELOP. \\
\hline 6.0 & 14 & TR. DELAHARE RIVER & BURLINGTON & FLORENCE & REALTY TRANSFER CO. \\
\hline 7.0 & 13 & IR. HAYNES CREEK & BURLINGTON & MEDFORD LAKES & MEDFORD LAKES COLONY CLUB \\
\hline 1.0 & 7 & TR. NO. BRANCH PENNSAUKEN & BURLINGTON & MAPLE SHADE & TUP. MOORESTOWN \\
\hline 0.0 & 15 & TR. NORTH RUN & BURLINGTON & NEW EGYPT & MICHAEL LUBLICK \\
\hline 2.0 & 17 & TRIB. BARTON RUN & BURLINGTON & BRADDOCK MILL & KENILHORTH ASSOC. \\
\hline 0.0 & 13 & BARTON RUN & CAMDEN & KRESSON & HERMAN ADUCAT \\
\hline 7.0 & 7 & BLUE ANCHOR BROOK & CAMDEN & SWEETWATER & BOB BENNETT \\
\hline 5.0 & 8 & BLUE ANCHOR BROOK & CAMDEN & SWEETWATER & BOB BENNETT \\
\hline
\end{tabular}




\begin{tabular}{|c|c|c|c|c|}
\hline 15 & COOPER RIVER & CAMDEN & CAMDEN & CAMDEN COUNTY \\
\hline 13 & COOPER RIVER & CAMDEN & HALLWORTH PARK & CAMBEN COUNTY \\
\hline 13 & COOPER RIVER & CAMDEN & SOMERDALE & MABEL. RICHMAN ESTATE \\
\hline 23 & COOPER RIVER & CAMDEN & HADDONFIELD & \\
\hline 7 & GREAT SWAMP BR. & CAMDEN & PLEASANT MILLS & REV CORPORATION \\
\hline 7 & HAYS MILL CREEK & CAMDEN & DUTCHTOHN & JOSEPH NARESSA \\
\hline 18 & NO. BRANCH BIG TIMBER CREEK & CAMDEN & CLEMENTON & CLEMENTON LAKE PARK INC. \\
\hline 12 & NO. BRANCH TIMBER CREEK & CAMDEN & CLEMENTON & \\
\hline 13 & NO. BRANCH TIMBER CREEK & CAMDEN & CLEMENTON & BOY SCOUTS OF AMERICA \\
\hline 30 & NORTH BRANCH TIMBER CREEK & CAMDEN & STRATFORD & BORO OF LAUREL SPRINGS \\
\hline 10 & PUMP BR. ALBERTSON BR. & CAMDEN & WINSLOW & \\
\hline 7 & PUMP BRANCH & CAMDEN & ANCORA & CAMP HALUWASA \\
\hline 8 & PUMP BRANCH & CAMDEN & ANCORA & CAMP HALUWASA \\
\hline 13 & SO. BR. TIMBER CREEK & CAMOEN & BLACKWOOD & CATHOLIC YOUTH \\
\hline 20 & SO. BRANCH NEWTON CREEK & CAMDEN & GLOUCESTER CITY & CAMDEN CITY PARK \\
\hline 13 & TIMBER CREEK SO. BRANCH & CAMDEN & GRENLOCK & PROVIDENT CORP. \\
\hline 6 & TR. MILLARD CREEK & CAMDEN & GIBBSBORO & A. CASSELMAN \\
\hline 9 & TRIB. BARTON RUN & CAMDEN & KRESSEN & CONNECTICUT CORP. \\
\hline 7 & WILDCAT BRANCH & CAMDEN & DUTCHTOWN & METCH CORP. \\
\hline 8 & BOG BRANCH CREEK & CAPE MAY & BEESLEY'S PT. & STATE OF NJ \\
\hline 10 & DEAN BRANCH & CAPE MAY & NORTH DENNIS & DEPT. OF CONSERV. \& DEVELOP. \\
\hline 8 & MILL CREEK & CAPE MAY & MARSHALLVILLE & MRS. KITTIE KING \\
\hline 8 & MILL CREEK & CAPE MAY & MARSHALLVILLE & MRS. KITTIE KING \\
\hline 8 & MILL CREEK & CAPE MAY & SEA ISLE CITY & STATE OF NJ \\
\hline 14 & S.W. BRANCH RANCOCAS CREEK & CAPE MAY & LUMBERTON & MEDFORD HISTORICAL SOCIETY \\
\hline 9 & SAVAGES RUN & CAPE MAY & DENAIS & STATE OF NJ \\
\hline 10 & TR. MILL CREEK & CAPE MAY & MARSHALLVILLE & MRS. KITTIE KING \\
\hline
\end{tabular}




\begin{tabular}{|c|c|c|c|c|c|c|}
\hline DIAMOND LAKE DAM & 21.2 & 10 & HOSPITALITY BR. & GLOUCESTER & FOLSOM & CITY OF GLOUCESTER \\
\hline SPRUCE LAKE & 10.6 & 7 & HOSPITALITY BR. & GLOUCESTER & FOLSOM & TIMBER LAKES BEACH CLUB \\
\hline TIMBER LAKES DAM & 52.0 & 8 & HOSPITALITY BRANCH & GLOUCESTER & FOLSOM & BIRCHHOOD LAKES COLONY \\
\hline FRANKLINVILLE LAKE DAM & 11.6 & 9 & LITTLE EASE BR. MARURICE RIVER & GLOUCESTER & FRANKLINVILLE & GEO FABRIZIO \\
\hline HENDRICKSON MILL DAM & 18.0 & 15 & LITTLE TIMBER CREEK & GLOUCESTER & REPAUCO STATION & GLOUCESTER CITY \\
\hline BETHEL LAKE CLUB & 11.4 & 13 & MANTUA CREEK & GLOUCESTER & SEHELLL & AB PERO \\
\hline EWANS MILL DAM & 31.0 & 15 & MULLICA RIVER & GLOUCESTER & MULLICA & GLOUCESTER COUNTY \\
\hline JAGGARDS POND DAM & 7.0 & 12 & NORTH BRANCH TIMBER CREEK & GLOUCESTER & DEN LAKE & BORO OF PINE HILL \\
\hline ALGONKIN LAKE DAM & 10.4 & 15 & OLDMANS CREEK & GLOUCESTER & EHAN & ALGONKIN CLUB \\
\hline ALGONKEN LAKE \#1 DAM & 50.0 & 14 & OLDMANS CREEK & GLOUCESTER & HARR I SONVILLE & BANCROFT SCHOOL \\
\hline LAKE WADSWORTH DAM & 12.5 & 17 & PITMAN & GLOUCESTER & PITMAN & HADSWORTH CRESSE JR. \\
\hline SO JERSEY WATER SUPPLY CO. & 86.0 & 19 & RACCOON CREEK & GLOUCESTER & MULLICA HILL. & HARRISON TWP. \\
\hline LAKE GILMAN DAM & 61.0 & 10 & RACCOON CREEK & GLOUCESTER & EWAN & LAKE GILMAN, INC. \\
\hline IDLE ACRES LAKE DAM & 71.0 & 7 & REED BRANCH & GLOUCESTER & PORCHTOWN & IDLE ACRES LAKE CORP. \\
\hline MALAGA DAM & 35.2 & 11 & SCOTLAND RUN & GLOUCESTER & MALAGA & FRANKLIN THP. \\
\hline FRIES MILLL DAM & 61.0 & 10 & SCOTLAND RUN & GLOUCESTER & FRANKLINVILLE & GLOUCESTER COUNTY \\
\hline GRENLOCK LAKE DAM & 93.0 & 21 & SO. BRANCH TIMBER CREEK & GLOUCESTER & BLACKHOOD & CAMDEN-GLOUCESTER CTY \\
\hline BLACKWOOD LAKE DAM & 59.0 & 15 & TIMBER CREEK SOUTH BRANCH & GLOUCESTER & BLENHEIM & CAMDEN \& GLOUCESTER CO. \\
\hline CRYSTAL SPRING LAKE & 41.0 & 6 & TR. FOURMILE BROOK & GLOUCESTER & ANCORA & MONROE TWP. \\
\hline PROCHES MILL DAM & 76.0 & 20 & TR. OLDMANS CREEK & GLOUCESTER & SHARPTOWN & JAMES MARGROVE \\
\hline LAKE BASGALORE DAM & 70.0 & 19 & TR. RACOON CREEK & GLOUCESTER & SWEDESBORO & \\
\hline GLENSIDE LAKE DAM & 13.0 & 12 & TR. SOUTH BRANCH TIMBER CREEK & GLOUCESTER & GRENLOCK & DIOCESES OF CAMDEN \\
\hline SUNSET LAKE DAM & 60.0 & 8 & WHITEHALL BRANCH & GLOUCESTER & FOLSOM & BOROUGH OF MOUNTAIN LAKES \\
\hline VICTORY LAKES DAM \#2 & 52.0 & 6 & WHITEHALL BRANCH & GLOUCESTER & FOLSOM & : \\
\hline VICTORY LAKES DAM \#1 & 19.0 & 8 & WHITEHALL BRANCH & GLOUCESTER & DIAMOND LAKE & E.J. LEHMAN \\
\hline ARTHUR F. FORAN DAM & 17.0 & 26 & BR. ALEXAUKEN CREEK & HUNTERDON & LAMBERTVILLEE & ARTHUR J. FORAN \\
\hline J. SEWARD JOHNSON DAM & 52.0 & 32 & COLD BROOK & HUNTERDON & OLOWICK & J. L. JOHNSON \\
\hline
\end{tabular}




\begin{tabular}{|c|c|c|c|c|c|c|}
\hline LAUREL LAKE DAM & 27.1 & 13 & BUCKSHUTEM CREEK & CUMBERLAND & LAUREL LAKE & HARREN \& ARTHUR SMADBECK \\
\hline BEAVER DAM & 15.8 & 9 & BUCKSHUTEN CREEK & CUMBERLAND & LAUREL LAKE & LAUREL LAKES BEACH CLUB \\
\hline E. ROGER JONES DAM & 51.0 & 8 & BURNT MILL BRANCH & CUMBERLAND & VINELAND & E. ROGER JONES \\
\hline MEMORIAL PARK POND & 60.0 & 8 & CEDAR BRANCH & CUMBERLAND & EAST VINELAND & CITY OF VINELAND \\
\hline CEDAR LAKE DAM & 22.1 & 10 & CEDAR CREEK & CUMBERLAND & CEDARVILLE & LAWRENCE TWP. \\
\hline SUNSET LAKE DAM & 39.7 & 8 & COHANSEY RIVER & CUMBERLAND & BRIDGETON & CITY OF BRIDGETON \\
\hline SEELEY'S MILL DAM & 25.3 & 10 & COHANSEY RIVER & CUMBERLAND & UPPER DEERFIELD THP. & LAND HOLDERS INC. \\
\hline MARY ELMER LAKE DAM & 68.0 & 15 & COHANSEY RIVER & CUMBERLAND & BRIDGETON & CITY OF BRIDGETON \\
\hline CUMBERLAND POND DAM & 90.0 & 14 & MANUNUSKI RIVER & CUMBERLAND & CUMBERLAND & MCCORISTON \\
\hline MENANTICO DAM & 17.8 & 8 & MEMANTICO CREEK & CUMBERLAND & MILLVILLE & MENANTICO COLONY \\
\hline EAST LAKE DAM & 75.0 & 17 & MILL CREEKK & CUMBERLAND & BRIDGETON & MURBELK KNITTED FIBERS \\
\hline DAVIS MILL DAM & 92.0 & 18 & RACOON DITCH & CUMBERLAND & RAY SIDE & ARTHUR DAVIS \\
\hline JERICHO POND DAM & 31.0 & 14 & STOW CREEK & CUMBERLAND & GARRISON CORNER & HENRY B. STEWART \\
\hline NO NAME & 58.0 & 8 & TR-CUB SWAMP & CUMBERLAND & DIVIDING CREEK & MATTHEWS CONTRACTING CO. \\
\hline LUMMIS LAKE LOWER DAM & 53.0 & 8 & TR. CEDAR CREEK & CUMBERLAND & CEDARVILLE & CEDARVILLE CORP. \\
\hline HANKINS POND DAM & 30.0 & 9 & TR. MAURICE RIVER & CUMBERLAND & HILLVILLE & CITY of MILLVILLE \\
\hline ELK LAKE DAM & 65.0 & 10 & TR. MOUNCE CREEK & CUMBERLAND & GREENWICH & DUFFY ESTATE \\
\hline HOFFMAN MILL DAM & 35.0 & 6 & WEST CREEK & CUMBERLAND & MAURICE RIVER & RICKY HOFFMAN \\
\hline VERONA LAKE DAM & 60.0 & 16 & PEEKMAN RIVER & ESSEX & VERONA & COUNTY OF ESSEX \\
\hline CAMPBELL POND DAM & 42.1 & 15 & WEST BR. RAHWAY RIVER & ESSEX & MILLBURN & CITY OF ORANGE \\
\hline WARNER LAKE DAM & 26.0 & 21 & & GLOUCESTER & & BORO OF WENONAH \\
\hline KINCAIO LAKE DAM & 70.0 & 16 & BIG CLEMS RUN & GLOUCESTER & RULLICA HILL & \\
\hline KANDLES POND DAM & 44.0 & 9 & BRANCH OF MANTUA CREER & GLOUCESTER & SEWELL & WEST KANDLE \\
\hline ALCYON LAKE DAM & 66.0 & 15 & CHESTNUT BR. MANTUA CREEK & GLOUCESTER & PITMAN & GLASSBORO-GLOUCESTER \\
\hline ROUGH ACRES DAM & 37.0 & 16 & CHESTNUT BRANCH MANTUA CREEK & GLOUCESTER & PITMAN & DOHINIC MAGAZU \& SONS \\
\hline NARRATICON LAKE DAM & 4.9 & 15 & CHURCH RUN & GLOUCESTER & SWEDESBORO & BOROUGH OF SWEDESBORO \\
\hline EVANS POND DAM & 13.4 & 7 & COOPER RIVER & GLOUCESTER & CAMDEN & CAMDEN CITY PARK COMM. \\
\hline CRANES DAM & 55.7 & 9 & HOSPITALITY BR. & GLOUCESTER & FOLSON & FRANK CRANE \\
\hline
\end{tabular}




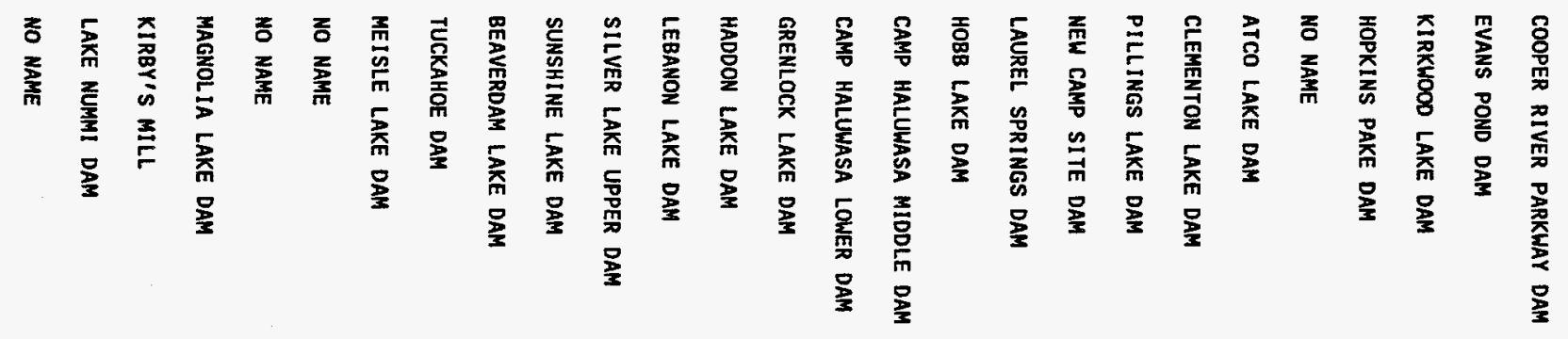

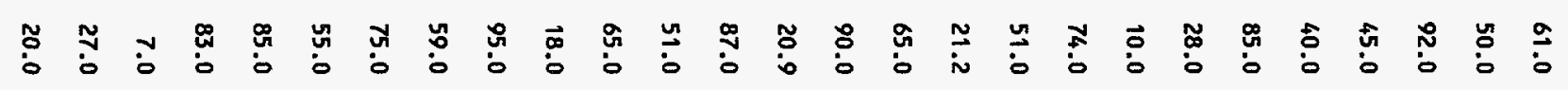

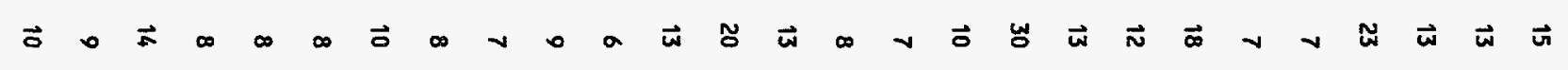

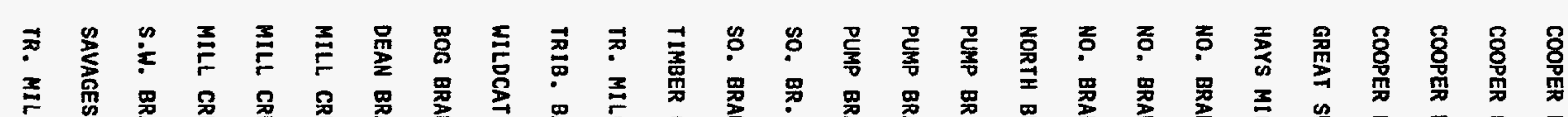

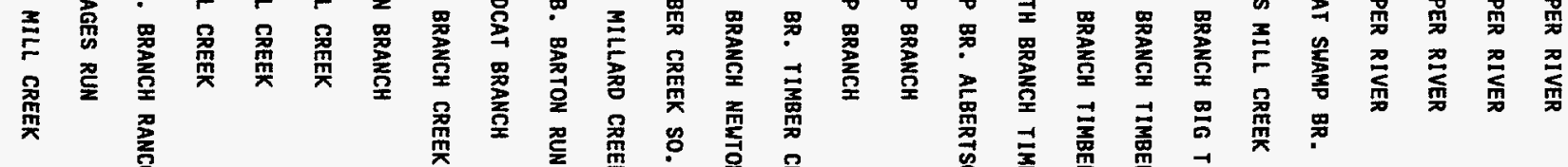

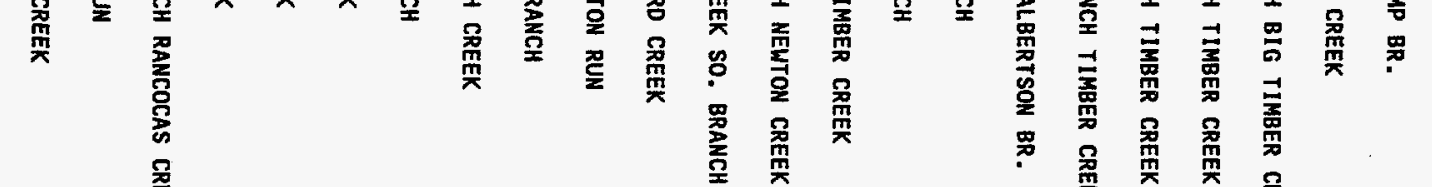
畨

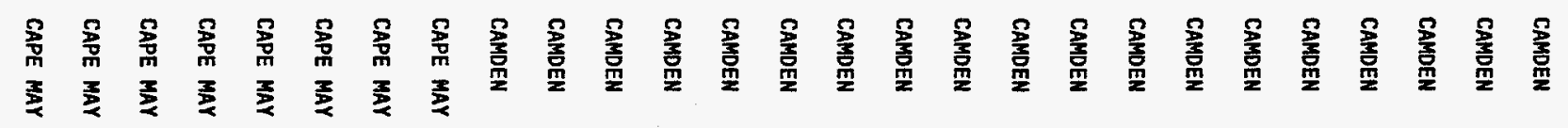

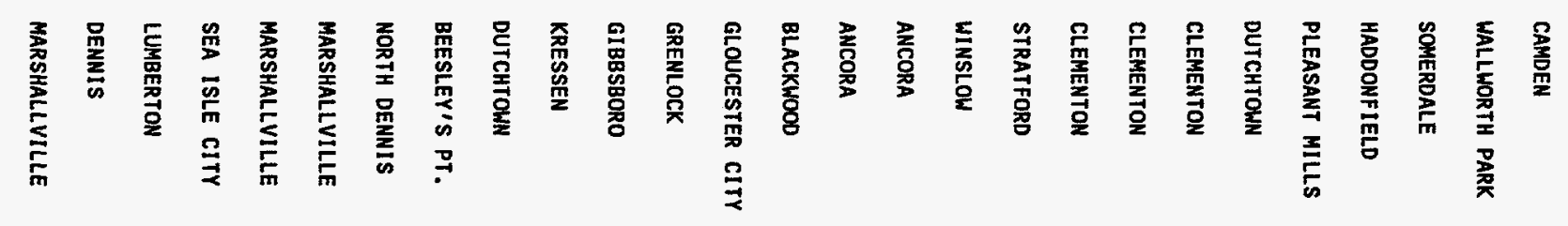

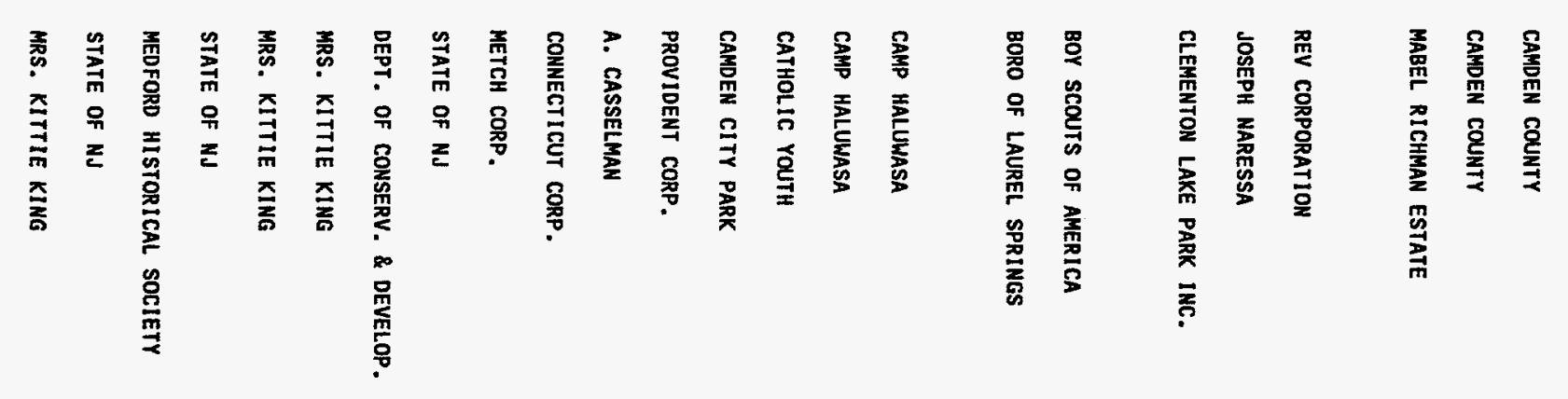




\begin{tabular}{|c|c|c|c|c|c|c|}
\hline BRADDOCKS MILL DAM & 9.0 & 16 & KETTLE RUN & BURLINGTON & TAUNTON LAKE & BRADDOCKS MILL \\
\hline KETTLE RUN CRENBERRY & 34.0 & 10 & KETTLE RUN & BURLINGTON & TAUNTON LAKE & MARLTON LAKES CIVIL ASSOC. \\
\hline SYLVAN LAKE DAM & 99.0 & 18 & MILL STREAM & BURLINGTON & BURLINGTON & CITY OF BURLINGTON \\
\hline NO NAME & 50.0 & 25 & MILL STREAM & BURLINGTON & BURLINGTON & BURLIHGTON TWP. \\
\hline NO NAME & 50.0 & 8 & MT. MISERY BRDOK & BURLINGTON & NEW LISBON & \\
\hline ATSION POND DAM & 97.8 & 7 & MULLICA RIVER & BURLINGTON & PLEASANT MILLS & STATE OF NJ \\
\hline AMPHIBIOUS LAKE DAM & 18.0 & 13 & NEWBOLD RUN & BURLINGTON & WRIGHTSTOWN & US ARMY \\
\hline SMITHVILLE DAM & 30.0 & 8 & NO. BR. RANCOCAS CR. & BURLINGTON & MT. HOLLY & CITY OF BURLINGTON \\
\hline MOORESTOWN W.W. DAM & 41.0 & 8 & NORTH BRANCH PENNSAUKEN RIVER & BURLINGTON & MOORESTOWN & TWP OF MOORESTOWN \\
\hline HARRISONVILLE DAM & 65.3 & 11 & OSWEGO RIVER & BURLINGTON & WADING RIVER & STATE OF NJ \\
\hline OSWEGO DAM & 70.8 & 10 & OSHEGO RIVER & BURLINGTON & MARTHA & STATE OF NS \\
\hline COUNTRY LAKES DAM \#1 & 21.9 & 12 & POLE BRIDGE BR. & BURLINGTON & NEW LISBON & COUNTY LAKES INC. \\
\hline LOWER DAM \#2 & 30.4 & 12 & POLE BRIDGE BR. & BURLINGTON & NEW LISBON & FRIENDSHIP LAKES, INC. \\
\hline COUNTY LAKE DAM \#2 & 35.4 & 14 & POLE BRIDGE BRANCH & BURLINGTON & NEW LISBON & COUNTRY LAKES INC. \\
\hline MIRROR LAKE DAM & 72.7 & 18 & RANCOCAS CREEK & BURLINGTON & BROWNS MILLS & PEMBERTON TWP. \\
\hline BIRCHWOOD LAKE DAM & 14.0 & 15 & SE BRANCH HAYNES CREEK & BURLINGTON & PINES LAKE & BIRCHWOOD LAKE COUNTRY CLUB \\
\hline NJ NO NAME & 50.0 & 15 & TO BLACK CREEK & BURLINGTON & CHESTERFIELD & WALTER GUZILOWSKI \\
\hline NO NAME & 85.0 & 8 & TO SOUTH RUN & BURLINGTON & MCGUIRE AFB & DOD USA \\
\hline NO NAME DAM & 80.0 & 15 & TO SOUTH RUN & BURLINGTON & MCGUIRE AFB & DOD USA \\
\hline LAKE ABSEGAMI DAM & 11.1 & 6 & TOMMY'S BROOK & BURLINGTON & NEW GRETNA & DEPT. OF CONS. \& DEVELOP. \\
\hline CRYSTAL LAKE DAM & 96.0 & 14 & TR. DELAWARE RIVER & BURLIMGTON & FLORENCE & REALTY TRANSFER CO. \\
\hline MISHE-MOKHA DAM & 27.0 & 13 & TR. HAYNES CREEK & BURLINGTON & MEDFORD LAKES & MEDFORO LAKES COLOHY CLUB \\
\hline STRAWBRIDGE LAKE UPPER DAM & 21.0 & 7 & TR. NO. BRANCH PENMSAUKEN & BURLINGTON & MAPLE SHADE & THP. MOORESTOWN \\
\hline NJ HO NAME & 70.0 & 15 & TR. NORTH RUN & BURLINGTON & NEW EGYPT & MICHAEL LUBLICK \\
\hline KENILWORTH LAKE DAM & 82.0 & 17 & TRIB. BARTON RUN & BURLINGTON & BRADDOCK MILL & KENIL.WORTH ASSOC. \\
\hline CEDAR LAKE DAM & 40.0 & 13 & BARTON RUN & CAMDEN & KRESSON & HERMAN ADUCAT \\
\hline NO NAME \#21 & 37.0 & 7 & BLUE ANCHOR BROOK & CAMDEN & SWEETWATER & BOB BENNETT \\
\hline NO NAME \#22 & 55.0 & 8 & BLUE ANCHOR BROOK & CAMDEN & SWEETWATER & BOB BENNETT \\
\hline
\end{tabular}




\begin{tabular}{|c|c|c|c|c|c|c|}
\hline NO NAME & 50.0 & 15 & MUSCONETCONG R. & HUNTERDON & HUGHESVI LLE & UNKNOWN \\
\hline ASBURY MILL DAM & 67.0 & 12 & MUSCONETCONG RIVER & HUNTERDON & BLOOMSBURY & ASBURY GRAPHITES \\
\hline FIMESVILLE MILL DAM & 17.0 & 9 & MUSCONETCONG RIVER & HUNTERDON & FINESVILLE & NOVERN INC. \\
\hline PENHELL MILL DAM & 37.0 & 9 & MUSCONETCONG RIVER & HUNTERDON & CHASGEHATER & ISABELLA THOMAS \\
\hline NISHISAKAHICK CREEK DAM & 71.0 & 24 & NISHISAKAWICK CREEK & HUNTERDON & EVERITTSTOWN & SALVATION ARMY \\
\hline DARTS MILL DAM & 57.0 & 14 & SO. BRANCH RARITAN RIVER & HUNTERDON & THREE BRIDGES & SVEN DANIELSON JR. \\
\hline LAKE CUSHETUNK DAM & 24.3 & 17 & SO. BRANCH ROCKAWAY CREEK & HUNTERDON & WHITEHOUSE & A. PEARSON \& J. APPLEBAUM \\
\hline CLINTON MILL DIKE & 21.0 & 8 & SOUTH BRANCH RARITAN RIVER & HUNTERDON & CLINTON & HUNTERDON ART CENTER \\
\hline ROCKFOLLOHS MILLS DAM & 37.0 & 9 & SOUTH BRANCH RARITAN RIVER & HUNTERDON & THREE BRIDGES & JACOB BIETZ \\
\hline CAMP REISTER DAM & 18.2 & 11 & SPRUCE RUN & HUNTERDON & GLEN GARDNER & NJ SYNOD LUTHERAN CHURCH \\
\hline CAMP BEISLER & 42.0 & 21 & SPRUCE RUN & HUNTERDON & GLEN GARDNER & NJ SYHOD LUTHERAN CHURCH \\
\hline SCHILLER POND DAM & 36.0 & 20 & TR. ALEXANKEN CREEK & HUNTERDON & MT. AIRY & SCHILLER \\
\hline AMWELL DAM \#1 & 50.0 & 20 & TR. ALEXAUKEN CREEK & HUNTERDON & LAMBERTVILLE & AMWELL REALTY \\
\hline AMHELL DAM \#2 & 69.0 & 19 & TR. ALEXAUKEN CREEK & HUNTERDON & LAMBERTVILLE & AMWELL VALLEY LAND CORP. \\
\hline NO NAME \#32 & 70.0 & 40 & TR. S. BRANCH RARITAN RIVER & HUNTERDON & SUNNYSIDE & \\
\hline STONY BROOK WS SITE \#11 & 41.0 & 22 & TR. STONY BROOK & HUNTERDON & LINVALE & HUNTERDON CITY \\
\hline POITERSVILLE RESERVOIR DAM & 26.0 & 24 & TRIB. LAMINGTON RIVER & HUNTERDON & POTTERSVILLE & ELIZABETHTOWN WATER CO. \\
\hline NJ NO NAME & 87.0 & 20 & BALDWIN CREEK & MERCER & PENNINGTON & MERCER CTY. \\
\hline GROVER MILL DAM & 37.8 & 8 & BEAR BROOK & MERCER & PRINCETOWN JCT. & C. L. DEY \\
\hline CEVA LAKE DAM & 45.0 & 14 & BRANCH SHABAKUNK CREEK & MERCER & EWING & TRENTON STATE COLLEGE \\
\hline BRAINERD LAKE DAM & 19.4 & 8 & CRANBURY BROOK & MERCER & PLAINSBORO & MIDDLESEX COUNTY \\
\hline RESEARCH FARM POND & 40.0 & 13 & HONEY BRANCH OF STONY BROOK & MERCER & HOPEWELL TWP. & STONY BROOK WATERSHED ASSOC. \\
\hline ASSUNPINK CREEK & 88.0 & 9 & LAWRENCE & MERCER & & GOODALL RUBBER CO. \\
\hline ASSUNPINK W.S. SITE \#8 DAM & 26.0 & 14 & POND RUN & MERCER & TRENTON & HAMILTON TWP. \\
\hline ETRA MILL DAM & 10.9 & 11 & ROCKY BROOK & MERCER & HIGHTSTOWN & A. KATZ \\
\hline MEMORIAL PARK DAM & 46.7 & 30 & ROCKY BROOK & MERCER & MERCER COUNTY & BORO OF BERNARDSVILLE \\
\hline PEDDIE LAKE DAM & 63.0 & 18 & ROCKY BROOK & MERCER & HIGHTSTOWN BOROUGH & BOROUGH OF HIGHTSTOWN \\
\hline COLONIAL LAKE DAM & 11.4 & 8 & SHABAKUNK CREEK & MERCER & TRENTON & COLONIAL OPERATING CO. \\
\hline
\end{tabular}




\begin{tabular}{|c|c|c|c|c|c|c|}
\hline PALMER LAKE & 50.0 & 10 & TO STONY BROOK & MERCER & PRINCETON & ELIZABETH GRANT CLARK \\
\hline STONY BROOK HATERSHED 4DAM & 79.6 & 20 & TR. STONY BROOK & MERCER & PRINCETON & STONY BROOK MILL \\
\hline STONY BROOK WS DAM \#7 & 33.0 & 34 & TR. STONY BROOK & MERCER & PENNINGTON & HUNT FAMILY \\
\hline SYLVA LAKE DAM & 16.0 & 22 & TR. STONY BROOK & MERCER & TRENTON & TRENTON STATE COLLEGE \\
\hline CREAM RIDGE DAM & 85.0 & 24 & TRIBUTARY TO CROSSWICK CREEK & MERCER & UPPER FREEHOLO TWP. & RUTGERS UNIVERSITY \\
\hline CREIGHTON LAKE DAM & 22.0 & 10 & AMBROSE BROOK & MIDDLESEX & MIDDLESEX & MIDDLESEX TUP. \\
\hline LAKE NELSON DAM & 40.0 & 11 & AMBROSE BROOK BRANCH RARITAN & MIDDLESEX & BOROUGH OF PISCATAWAY & LAKE MELSON IMPROVEMENT ASSOC. \\
\hline MIDDLESEX MILLING DAM & 42.2 & 8 & BOUND BROOK & MIDDLESEX & MIDDLESEX & MIDDLESEX MILLING \\
\hline DAVIDSON'S MILL POND DAM & 13.4 & 10 & LAUREMCE BROOK & MIDDLESEX & NO. BRUNSHICK & CITY OF MEW BRUNSWICK \\
\hline HILL POND DAM & 57.9 & 8 & LAWRENCE BROOK & MIDDLESEX & SAYREVILLE & \\
\hline DEANS MILLS DAM & 20.0 & 9 & LAHRENCE BROOK & MIDDLESEX & PATRICK CORNERS & BENJAMIN HEISENFIELD \\
\hline DEVOE LAKE DAM & 49.2 & 11 & MANALAPAN BROOK & MIDDLESEX & SPOT SWOOD & SPOTSHOOD BORO \\
\hline MANALAPAN LAKE DAM & 26.1 & 9 & MANALAPAN BROOK & MIDDLESEX & JAMESBURG & MIDDLESEX DEPT. OF PARKS \\
\hline HELMETTA DAM & 68.0 & 7 & MANALAPAN BROOK & MIDDLESEX & HELMETTA & MIDDLESEX CTY. \\
\hline NO NAME & 52.0 & 12 & SLOPE BROOK & MIDDLESEX & LINCROFT & STAVOLA BROS. \& ORGO FARM \\
\hline ROOSEVELT PARK DAM & 26.0 & 8 & SO. BRANCH RAHWAY RIVER & MIDDLESEX & WOODBR IDGE & COUNTY OF MIDDLESEX \\
\hline LOUISE LAKE DAM & 65.0 & 10 & & MONMOUTH & & LAKE LOUISE COMM. CLUB. \\
\hline ASSUNPINK DAM SITE \#4 & 12.5 & 18 & ASSUNPINK CREEK & MONMOUTH & HINDSOR & STATE OF NJ \\
\hline MARLU LAKE DAM & 42.0 & 27 & BRANCH OF HOP BROOK & MONMOUTH & RED BANK & MAURICE POLLACK \\
\hline FRANKLIN LAKE DAM & 70.0 & 7 & BRANCH TURTLE MILL BROOK & MONMOUTH & LONG BRANCH & TOWN OF MEST LONG BRANCH \\
\hline ALDRICH LAKE DAM & 58.0 & 9 & BUCKS BRANCH & MONMOUTH & LAKEWOOD & CHARLES ALDRICH \\
\hline IMLAYSTOWN LAKE DAM & 18.4 & 19 & DOCTORS CREEK & MONMOUTH & IMLAYSTIOWN & STATE OF NJ \\
\hline RED VALLEY DAM & 50.0 & 11 & DOCTORS CREEK & MONMOUTH & IMLAYSTOWM & MONMOUTH СTY. \\
\hline INDIAN LAKE DAM & 10.0 & 19 & INDIAN RUN & MONMOUTH & UPPER FREEHOLD & MONMOUTH CTY. \\
\hline JUMPING BROOK DAM & 60.0 & 10 & JUMPING BROOK & MONMOUTH & SO. BELMAR & MONMOUTH COUNTY UATER CO. \\
\hline ZENNIC W.S. & 61.0 & 14 & MANALAPAN BROOK & MONMOUTH & MILLHURST & DAVID R. BULKK \\
\hline
\end{tabular}




\begin{tabular}{|c|c|c|c|c|c|c|}
\hline MILLHURST DAM & 92.0 & 14 & MANALAPAN BROOK & MONMOUTH & MILLHURST & MILLHURST MILLS \\
\hline ALLAIRE DAM & 62.0 & 12 & MILL RUN OF MANASQUAN RIVER & MONMOUTH & ALLAIRE STATE PARK & MONMOUTH CTY. \\
\hline NO NAME DAM & 60.0 & 10 & NEGRO RUN & MONMOUTH & ALLENTOWN & HARRY KNOWLES, SR. \\
\hline NO NAME DAM & 55.0 & 10 & POLLY POD BROOK & MONMOUTH & SOUTH BELMAR & MRS REALYY \\
\hline PORICY DAM & 65.0 & 7 & PROICY BROOK & MONMOUTH & RED BANK & MONMOUTH CTY. \\
\hline SHADOW LAKE DAM & 11.2 & 15 & QUIGLEY'S CREEK & MONMOUTH & RED BANK & COUNTY OF MONMOUTH \\
\hline PERRINEVILLE DAM & 36.0 & 17 & ROCKY BROOK & MONMOUTH & PERRINEVILLE & MONMOUTH CTY. \\
\hline NJ NO NAME & 56.0 & 6 & TO MANASQUAN RIVER & MONMOUTH & WYCKOFF MILLS & MONMOUTH CO. PARKS DEPT. \\
\hline NO NAME DAM & 60.0 & 10 & TO ROCKY BROOK & MONHOUTH & EAST HINDSOR & LOUIS E + M. C. DIVITIS \\
\hline ASSUNPINK DAM SITE \#5 & 0.0 & 9 & TR. ASSUNPINK CREEK & MONMOUTH & UINDSOR & STATE OF NJ \\
\hline EATONTOWN & 60.0 & 10 & WAMPUM & MONMOUTH & & EASTON PARKS COMM. \\
\hline ENGLISHTOWN LAKE DAM & $\mathbf{5 8 . 0}$ & 13 & WEAMACONK CREEK & MONMOUTH & ENGLISHTOWN & MONMOUTH CTY. \\
\hline WEMROCK POND DAM & 58.0 & 12 & HEMROCK CREEK & MONMOUTH & ENGLISHTOWN & \\
\hline OSBORNS MILLS DAM & 86.0 & 12 & WRECK POND BROOK & MONMOUTH & SPRING LAKE HEIGHTS & T. H. BENNETT \\
\hline OLD MILL POND DAM & 35.0 & 9 & WRECK POND BROOK & MONHOUTH & SEA GIRT & WALL TWP. \\
\hline HURLEYS POND & $\mathbf{5 2 . 0}$ & 6 & WRECK POND BROOK & MONMOUTH & ALGER MILLS & W. D. + G. H. MAUDSLEY \\
\hline BUCKS MILL DAM & 14.6 & 14 & YELLOW BROOK & MONMOUTH & RED BANK & COUNTY OF MONMOUTH \\
\hline SPLIT ROCK POND DAM & 23.0 & 38 & BEAVER BROOK & MORRIS & DENVILLEE & CITY OF JERSEY CITY \\
\hline SUNSET LAKE DAM & 74.0 & 8 & BOROUGH TROY BROOK & MORRIS & BOROUGH OF MOUNTAIN LAKES & BOROUGH OF MOUNTAIN LAKES \\
\hline SIEBERT POND DAM & 42.0 & 20 & BR. BEAVER BROOK & MORRIS & DENVILLE & NJ CAMP BLIND CHILDREN \\
\hline CHEROKEE LAKE DAM & 16.0 & 11 & BR. INDIA BROOK & MORRIS & MENDHAM & ARCHDIOCOSE OF PATERSON \\
\hline NJ NO NAME \#10 DAM & 33.0 & 8 & BRANCH OF BEAVER BROOK & MORRIS & DENVILLE & SHIRE NAT'L. CORP. \\
\hline EGBERT RESORT LAKE DAM & 46.0 & 11 & BURNT MEADOW BROOK & MORRIS & PICATINNY ARSENAL & KENMETH EGBERT \\
\hline INDIAN LAKE DAM & 8.8 & 12 & DEN BROOK & MORRIS & DENVILLE & INDIAN LAKE COMMUNITY CLUB \\
\hline ESTLING & 5.6 & 7 & DEN BROOK & MORRIS & DENVILLE & NJDOT \& ESTLING \\
\hline OPENAKA LAKE DAM & 29.0 & 16 & OEN BROOK & MORRIS & DENVILLE & ROBERT PRICE \\
\hline GEORGE LAKE DAM & 69.0 & 30 & ELECTRIC BROOK & MORRIS & LONG VALLEY & MORRIS COUNTY PARKS COMM. \\
\hline FOXS LAKE DAM & 63.0 & 22 & FOXS BROOK & MORRIS & BOROUGH OF ROCKAWAY & ROCKAWAY THP. \\
\hline
\end{tabular}




\begin{tabular}{|c|c|c|c|c|c|c|}
\hline EMMA LAKE & 72.0 & 10 & HIBERNIA BR. & MORRIS & HIBERNIA & DR. ANTONIO \\
\hline NO NAME & 56.0 & 10 & HIBERNIA BROOK & MORRIS & HIBERNIA & \\
\hline LAKE TELEMARK DAM & 70.0 & 11 & HIBERNIA BROOK & MORRIS & HIBERNIA & L. TEL COUNTRY CLUB \\
\hline NO NAME & 80.0 & 10 & LAMINGTON RIVER & MORRIS & MENDHAM & UNKNOWN \\
\hline NO NAME \#3388 & 66.0 & 9 & MALAPARDIS BROOK & MORRIS & WHIPPANY & UHIPPANY PAPER BOARD CO. \\
\hline CROMWELL LAKE DAM & 27.0 & 13 & MCVICKERS BROOK & MORRIS & PEAPACK GLADSTONE & JOHN LUNDBERG \\
\hline DALRYMPLE POND & 39.0 & 14 & MILL BROOK & MORRIS & RANDOLPH IWP. & COUNTY COLLEGE OF MORRIS \\
\hline MINE HILL RESERVOIR DAM & 34.0 & 25 & MINE BROOK & MORRIS & HACKETTSTOWN & HACKETTSTOWN \\
\hline LAKE MUSCONETCONG DAM & 33.0 & 10 & MUSCONETCONG RIVER & MORRIS & NETCONG & STATE OF NJ \\
\hline LAKE WATERLOO DAM & 68.0 & 9 & MUSCONETCONG RIVER & MORRIS & HACKETTSTOWN & STATE OF NJ \\
\hline LAKE HOPATCONG DAM & 44.7 & 16 & MUSCONETCONG RIVER & MORRIS & NETCONG & STATE OF NJ \\
\hline LAKE THERESE DAM & 92.4 & 30 & NO. BR. RARITAN R. & MORRIS & PEAPACK & AT\&T \\
\hline JAGUI MILL DAM & 13.7 & 16 & NO. BR. WHIPPANY $R$. & MORRIS & MORRISTOWN & GREYSTONE PRK PSYCHIATRIC HOSP \\
\hline WASHINGTON CORNER DAM & 34.0 & 12 & PASSAIC RIVER & MORRIS & BERNARDSVILLE & MORRIS COUNTY PARK COMM. \\
\hline COLD SPRING LAKE DAM & 2.1 & 13 & PEQUANNOCK R. & MORRIS & BUTLER & SALVATION ARMY \\
\hline MT. KIMBALL LAKE DAM & 76.6 & 29 & PRIMROSE BROOK & MORRIS & BASKING RIDGE & LAKESHORE CORP. \\
\hline WASHINGTON FORGE POND DAM & 32.0 & 10 & ROCKAMAY R. & MORRIS & WHARTON & BOROUGH OF WHARTON \\
\hline OLD MORRIS CANAL DAM & 27.0 & 18 & ROCKAWAY RIVER & MORRIS & BOONTON & UNKNOWN \\
\hline POWERVILLLE DAM & 17.0 & 12 & ROCKAWAY RIVER & MORRIS & BOONTON & JOHN ROEMER \\
\hline NO NAME DAM & 90.0 & 18 & SO. BR. RARITAN RIVER & MORRIS & BARTLEY & \\
\hline KIKEOUT RESERVOIR DAM & 42.0 & 15 & STONE HOUSE BROOK & MORRIS & BUTLER & BORO OF KINNELON \\
\hline No NAME & 63.0 & 13 & TO ELECTRIC BR. & MORRIS & LONG VALLEY & HASHINGTON TWP: \\
\hline COOKS POND & 92.0 & 6 & TO ROCKAWAY RIVER & MORRIS & DENVILLE & F. L. PETROZZZ \\
\hline SUNRISE LAKE & 50.0 & 18 & TO UHIPPANY RIVER & MORRIS & WASHINGTON VALLEY & MORRIS CITY PARKS COMM. \\
\hline NO NAME & 90.0 & 20 & TO HHIPPANY RIVER & MORRIS & MORRISTOWN & \\
\hline
\end{tabular}




\begin{tabular}{|c|c|c|c|c|c|c|}
\hline POWOER MILL POND & 90.0 & 40 & TO WHIPPANY RIVER & MORRIS & MORRIS PLAINS & TABOR LAKE CORP. \\
\hline LAKE ARROHHEAD DAM & 96.0 & 8 & TR. DEN BROOK & MORRIS & DENVILLE & LAKE ARROWHEAD CLUB \\
\hline SAMMILL POND DAM & 28.0 & 27 & TR. EAST DITCH & MORRIS & PEQUANHOCK & JOSEPH MCDERMOTT \\
\hline TERRACE LAKE DAM & 11.0 & 7 & TR. PEQUANNOCK RIVER & MORRIS & BUTLER & CHRISTIAN RECREATIONAL ASSOC. \\
\hline KINNELON (MAPLE) DAM & 25.0 & 14 & TR. PEQUANMOCK RIVER & MORRIS & BUTLER & COLUMBIA SAVING \& LOAN \\
\hline DIXONS POND DAM & 86.0 & 8 & TR. ROCKAHAY RIVER & MORRIS & POWERVILLE & DIXON ASSOC. \\
\hline LAKE MORSKI OKO DAM & 54.0 & 12 & TR. ROCKAWAY RIVER & MORRIS & MILTON & MORSKIE OKO INC. \\
\hline ALOHA LAKE DAM & 19.6 & 21 & TR. WHIPPANY RIVER & MORRIS & MORRISTOWN & MRS. C. HUTCHINSON \\
\hline DECOZEN'S DAM & 38.0 & 11 & TROY BROOK & MORRIS & PARSIPPANY - TROY HILLS & INTERPACE CORP. \\
\hline BIRCHWOOD LAKE DAM & 89.0 & 20 & TROY BROOK & MORRIS & MOUNTAIN LAKES & BOROUGH OF MOUNTAIN LAKES \\
\hline NO NAME \#30 & 50.0 & 15 & WALDON BROOK & MORRIS & HUROTOWN & \\
\hline SWANNANDA LAKE DAM \#1 & 21.4 & 14 & WEST BR. ROCKAWAY R. & MORRIS & WOODSTOCK & RINGLING MANOR \\
\hline SWANNANDA LAKE DAM \#2 & 11.4 & 8 & WEST BR. ROCKAWAY R. & MORRIS & WOODSTOCK & RINGLING MANOR \\
\hline POCAHONTAS DAM & 30.7 & 11 & WHIPPANY R. & MORRIS & MORRISTOWN & TOWN OF MORRISTOWN \\
\hline SPEEDWELL DAM & 33.3 & 12 & WHIPPANY R. & MORRIS & MORRISTOWN & TOWN OF MORRISTOWN \\
\hline MORRISTOWN RESERVOIR DAM & 13.1 & 54 & WHIPPANY RIVER & MORRIS & BROOKSIDE & MORRISTOWN \\
\hline EDEN MILL DAM & 83.0 & 14 & WHIPPANY RIVER & MORRIS & HANOVER & WHIPPANY PAPER BOARD \\
\hline MT. HOPE LAKE DAM & 2.3 & 11 & WHITE MEADOH BR. & MORRIS & ROCKAWAY TWP. & HALECREST CORP. \\
\hline WHITE MEADOW LAKE DAM & 4.5 & 14 & WHITE MEADOW BROOK & MORRIS & ROCKAWAY & WHITE MEADOW LAKE PROP. OWNERS \\
\hline CASSVILLE DAM & 65.0 & 8 & BRANCH OF TOMS RIVER & OCEAN & CASSVILLE & ROVA FARMS SCHOOL \\
\hline DOUBLE TROUBLE DAM & 43.1 & 7 & CEDAR CREEK & OCEAN & LANOKA HARBOR (LACEY TWP.) & STATE OF NJ \\
\hline OAK FORD LAKE DAM & 29.5 & 7 & CROSSWICKS CREEK & OCEAN & NEW EGYPT & PLUMSTED TUP. \\
\hline WRIGHTS DAM & 65.0 & 12 & DAVENPORT BR. TOMS RIVER & OCEAN & TOWS RIVER & TWP OF MANCHESTER \\
\hline HOLIDAY LAKE DAM & 21.0 & 19 & FOUR MILE BRANCH & OCEAN & MANAHAWKIN & OCEAN ACRES \\
\hline HANOVER DAM & 51.0 & 8 & GAUNTS BROOK & OCEAN & BROWNS MILL. & STATE OF NJ US ARMY \\
\hline PAHATCONG LAKE DAM & 87.0 & 9 & GIFFORDS MILL B. TUCKERTON CK. & OCEAN & TUCKERTON & Dor \\
\hline RAINBOW LAKE DAM & 55.0 & 13 & KETTLE CREEK & OCEAN & CEDARWOOD PARK & LAKE RIVIERA \\
\hline LAKE RIVIERA DAM & 37.0 & 11 & KETTLE CREEK & OCEAN & CEDARHOOD PARK & LAKE RIVIERA \\
\hline
\end{tabular}




\begin{tabular}{|c|c|c|c|c|c|c|}
\hline PROSPERTOWN DAM & 5.1 & 14 & LAHAWAY CREEK & OCEAN & UPPER FREEHOLD THP. & STATE OF NJ \\
\hline GLIDDON LAKE DAM & 70.0 & 14 & LONG BROOK & OCEAN & WHITESVILLE & CLAYTON SAND CO. \\
\hline HO NAME \#23 & 90.0 & 7 & MANAPAQUE BROOK & OCEAN & LAKEHURST & \\
\hline MANAHAWKIN LAKE DAM & 21.0 & 10 & MILL CREEK & OCEAN & CEDAR RUN & OCEAN COUNTY \\
\hline MILL DAM & 22.0 & 12 & OYSTER CREEK & OCEAN & WELLS MILLS & BOY SCOUTS OCEAN CO. \\
\hline NO NAME DAM & 54.0 & 10 & SHANE BROOK & OCEAN & LAKEHURST N.A. & STATE OF NJ \\
\hline LAKE SUCCESS DAM & 51.5 & 9 & SHANNAE BROOK & OCEAN & LAKEHURST NAVAL AIR ST. & STATE OF NJ \\
\hline BENNETS MILLS DAM & 28.3 & 14 & SO. BR. METEDECONK RIVER & OCEAN & JACKSON TWP. & EDWARD ENNO \\
\hline LAKE SHENANDOAH & 44.7 & 14 & SO. BRANCH METEDECONK RIVER & OCEAN & BRICKTOWN & OCEAN COUNTY \\
\hline JACKSONS MILLS DAM & 95.0 & 15 & SO. BRANCH METEDECONK RIVER & OCEAN & JACKSON MILLS & SAMBOL CONSTR. CO. \\
\hline LAKE HORICON DAM & 27.0 & 9 & UNION BRANCH & OCEAN & TOMS RIVER & BOROUGH OF LAKEHURST \\
\hline STAFFORD FORGE DAM & 22.6 & 10 & WESTECUNK CREEK & OCEAN & WEST CREEK & JAMES STAFFORD \\
\hline UPPER KESWICK DAM & 66.0 & 15 & WRANGEL BROOK & OCEAN & KENHICH GROVE & AMERICAN KESWICH \\
\hline WONDER LAKE DAM & 51.0 & 7 & APSHAWA BROOK & PASSAIC. & BUTLER & WONDER LAKE PROPERTY OWNER \\
\hline PINECLIFF LAKE DAM & 12.8 & 16 & BELCHER CREEK & PASSAIC & WEST MILFORD & PINECLIFF LAKE COMM. CLUB \\
\hline PINECREST DAM & 25.0 & 18 & BELCHER CREEK & PASSAIC & WEST MILFORD & JOHN GLUECK \\
\hline HARRISON MOUNTAIN LAKE DAM & 76.0 & 16 & BR. BURNT MEADOW BROOK & PASSAIC & STONETOWN & L\&Z REALTY COMPANY \\
\hline UPPER TWIN LAKES DAM & 48.0 & 10 & BR. DOSTS BROOK & PASSAIC & POMPTON LAKES & KEARFOTT-DIV. OF SINGER \\
\hline RICKONDA LAKE DAM & 46.0 & 15 & BR. HANAQUE RIVER & PASSAIC & MONKS & LAKE RICKONDA RESORT INC. \\
\hline LAKE SONOMA DAM & 74.0 & 24 & BURNT MEADOW BROOK & PASSAIC & STONETOWN & TAPAUINGO TROUT PRESERVE \\
\hline CLINTON RESERVOIR DAM & 20.8 & 18 & CLINTON BROOK & PASSAIC & NEWFONNOLAND & CITY OF NEWARK \\
\hline CUPSAW LAKE DAM & 14.3 & 31 & CUPSAH BROOK & PASSAIC & ERSKIME & CUPSAW LAKE IMP. ASSOC. \\
\hline PINES LAKE DAM & 18.5 & 40 & HAYCOCK BROOK & PASSAIC & PUMPTON LAKES & PINES LAKE ASSOC. \\
\hline POINTVIEW DAM & 12.1 & 61 & HAYCOCK BROOK & PASSAIC & WAYNE & PASSAIC VALLEY \\
\hline LIONSHEAD LAKE DAM & 60.0 & 28 & HAYCOCK BROOK & PASSAIC & WAYNE & WAYNE TUP. \\
\hline IRISH BROOK DAM & 4.0 & 10 & IRISH BROOK & PASSAIC & BLOOMINGDALE & LAKE IOSCO CORP. \\
\hline MORSE LAKES DAM & 61.0 & 14 & IRISH BROOK & PASSAIC & POMPTON LAKES & MORSE LAKES PROPERTY \\
\hline
\end{tabular}




\begin{tabular}{|c|c|c|c|c|c|c|}
\hline UPPER GREENWOOD DAM & 10.9 & 6 & LONG HOUSE CREEK & PASSAIC & BELLRALE, NY & GREENWOOD LAKE PROPERTY \\
\hline WEST MILFORD LAKE DAM & 46.0 & 16 & LONG HOUSE CREEK & PASSAIC & WEST MILFORD & WEST MILFORD COMMUNITY \\
\hline LOOKOVER LAKE DAM & 40.0 & 12 & LONGHOUSE BROOK & PASSAIC & GREENWOOD & LAKE LOOKOVER DEVEL. CORP. \\
\hline OLDHAM POND DAM & 12.6 & 22 & MOLLY ANNS BROOK & PASSAIC & HALEDON & HARMON COLOR WORKS \\
\hline CARPI LAKE DAM & 37.0 & 9 & MORSETOWN BROOK & PASSAIC & WEST MILFORD & HELEN \& FAUST JAFRATE \\
\hline BEARFORT WATERS DAM & 60.0 & 12 & MOSEMANNS BROOK & PASSAIC & CLINTON RESERVOIR & STATE OF NJ \\
\hline GLEN WILD LAKE DAM & 1.7 & 15 & MUD RUN & PASSAIC & BLOOMINGDALE & GLENWILD LAKE ASSOC. \\
\hline BEATTIES MILL DAM & 65.0 & 9 & PASSAIC RIVER & PASSAIC & SINGAC & PASSAIC VALLEY WATER COMM. \\
\hline WASHINGTON LAKE DAM & 48.0 & 11 & POST BROOK & PASSAIC & HASKELL & WANAQUE TWP. \\
\hline ZELIFF POND DAM & 30.0 & 10 & POSTS BROOK & PASSAIC & POMPTON LAKES & JAMES \& MARY HOSFORD \\
\hline RINGWOOD MILL DAM & 23.1 & 11 & RINGHOOD CREEK & PASSAIC & ERSKINE & DEP \\
\hline SKYLINE LAKE DAM \#2 & 6.1 & 20 & SHEPARD BROOK & PASSAIC & WANAQUE & SKYLINE LAKE PROP. OWNERS \\
\hline SKYLINE LAKE DAM \#1 & 85.0 & 16 & SHEPHERD BR PASSAIC RV BASIN & PASSAIC & BOROUGH OF WANAQUE MIDVALE & SKYLINE LAKE PROP OWNER ASSOC. \\
\hline HIGHLAND LAKE DAM & 49.0 & 40 & SLIPPERY ROCK BROOK & PASSAIC & WEST PATTERSON & BANK OF NS \\
\hline WALDRICK BLEACHERY DAM & 10.6 & 8 & THIRD RIVER & PASSAIC & NUTLEY & WALDRICK BLEACHERY \\
\hline GORDON LAKES DAH & 46.0 & 12 & TR. POST BROOK & PASSAIC & BLOOMINGDALE & \\
\hline BARBOUR NILL DAM & 50.0 & 8 & TR. PREAKNESS BROOK & PASSAIC & HAYNE TUP. & GARY VAN DECKER \\
\hline NOSENGO POND DAM & 40.0 & 9 & TR. WEST BROOK & PASSAIC & WEST MILFORD & CATHOLIC YOUTH ORGANIZATION \\
\hline KAMPFE LAKE DAM & 93.0 & 11 & TRIB. PEQUANOCK RIVER & PASSAIC & BUTLER & KAMPFE LAKE ASSOC. \\
\hline HARRISON MT. LAKE & 21.0 & 10 & WANAQUE RESERVOIR & PASSAIC & WANAQUE & 12 REALTY CORP. \\
\hline KITCHELL DAM & 18.2 & 14 & WEST BROOK & PASSAIC & WANAQUE & WARREN KITCHELL \\
\hline LOWER MT. GLEM LAKE DAM & 19.5 & 15 & WEST BROOK & PASSAIC & WANAQUE & MT. GLEN LAKES ASSOC. \\
\hline LINDY'S LAKE DAM & 29.9 & 23 & WEST BROOK & PASSAIC & WANAQUE & LINDY'S LAKE \\
\hline UPPER MOUNT GLEN LAKE DAM & 28.0 & 10 & WEST BROOK & PASSAIC & WEST MILFORD TWP. & MOUNT GLEN LAKES ASSOC. \\
\hline ALLOWAY LAKE DAM & 31.0 & 13 & ALLOWAY CREEK & SALEM & ALLOWAY & MCELDOWNEY CO. \\
\hline BALLINGERS MILL POND DAM & 81.0 & 23 & COOL RUN & SALEM & ALLOWAY & SALEM CITY \\
\hline ROOSEVELT CAMP DAM & 60.0 & 15 & COOL RUH & SALEM & ALLOWAY & SALEM CTY. \\
\hline WENTZELL-WILSON DAM & 36.0 & 18 & COOL RUA & SALEM & ALLOWAY & HERB WENTZELL \\
\hline
\end{tabular}




\begin{tabular}{|c|c|c|c|c|c|c|}
\hline THUNDERGUST POND DAM & 60.0 & 10 & DEEP RUN & SALEM & QUINTON & MR. ESPOSITO \\
\hline COBBS MILL DAM & 29.0 & 11 & DEEP RUN & SALEM & PARADISE LAKES & SALEM COUNTY \\
\hline NICKLES MILL DAM & 61.0 & 15 & HORSE RUN & SALEA & JERICKO & REALTY DEWILDES RHOOO \\
\hline QUINTON POND DAM & 40.0 & 8 & LAUREL RUN & SALEM & QUINTON & \\
\hline CENTREDON LAKE DAN & 39.5 & 9 & RUDDY RUN & SALEM & CENTREDON & CENTREDON LAKES PARK ASSN. \\
\hline HANSON DAM & 39.5 & 8 & SALEM CANAL & SALEM & & E.I. DUPONT DE NEMOURS \\
\hline BROWN DAM & 66.0 & 10 & SALEM CREEK & SALEA & DEEPUATER & E.I. DUPONT DE NEMOURS \\
\hline CAMP KARNEY DAM & 14.3 & 11 & SALEM CREEK & SALEM & WOODSTOWN & YMCA-SALEM CO. \\
\hline EAST LAKE DAM & 72.0 & 11 & SALEM CREEK & SALEM & WOODSTOWN & \\
\hline DARETOWN LAKE & 86.0 & 16 & SALEM R. & SALEM & WOODSTOWN & UPPER PITTSGROVE TUP. \\
\hline VETERANS MEMORIAL LAKE DAM & 88.0 & 14 & SALEM RIVER & SALEM & WOODSTOWN & TOWN OF WOODSTOWN \\
\hline SADIES DAM & 36.0 & 14 & SALEM RIVER & SALEM & WOODSTON & ABE COTLER \\
\hline THUNDERGUST POND DAM & 41.0 & 13 & THUNDERGUST BROOK & SALEM & MILLVILLE & STATE OF NJ \\
\hline NO NAME & 52.0 & 8 & TR. ALLLOWAY & SALEM & HANCOCKS BRIDGE & DAVID A. FOGG \\
\hline COXHAT POND DAM & 52.0 & 9 & TR. DEEP RUN & SALEM & QUINTON & PIERCE HAMMOND \\
\hline NO NAME DAM \#37 & 16.0 & 18 & BRANCH BEOEN BROOK & SOMERSET & ROCKY HILL & MACPHERSON RAYMOND \\
\hline SUNSET LAKE DAM & 34.0 & 16 & CHAMBERS BROOK & SOMERSET & BEDMISTER & \\
\hline EAST BRANCH RESERVOIR DAM & 60.0 & 21 & MIDDLE BROOK & SOMERSET & BOUND BROOK & ELIZABETHTOWN WATER \\
\hline RAVINE LAKE DAM & 89.4 & 34 & NORTH BR. RARITAN RIVER & SOMERSET & FAR HILLS & RAVINE ASSOC. \\
\hline VAN OORANS MILL DAM & 16.4 & 17 & PASSAIC RIVER & SOMERSET & BASKING RIDGE & JOHN ZIEBARTH \\
\hline SKILLMAN DAM & 25.0 & 16 & ROCK BROOK & SOMERSET & GRIGGSTOWN & STATE OF MJ \\
\hline SPOOKY BROOK DAM & 92.0 & 21 & TR. DELAWARE RARITAN CANAL & SOMERSET & EAST MILLSTONE & SOMERSET CO. PARK COHM. \\
\hline SAWMILL POND DAM & 70.0 & 14 & BIG FLAT BROOK & SUSSEX & LAKE OCQUITTANK & STATE OF NJ \\
\hline SKELLINGER DAM & 98.0 & 11 & BIG FLAT BROOK & SUSSEX & WALLPACK & STATE OF NJ \\
\hline GLENHOOD LAKE DAM & 56.0 & 12 & BLACK CREEK & SUSSEX & MCAFEE & BROWN \& BAILEY MILK CO. \\
\hline SWARTSHOOD LAKE DAM & 20.8 & 11 & BR. OF PAULINS KILL & SUSSEX & MIDDLEVILLE & NJ DIV PARKS \& FORESTRY \\
\hline LAKE GRINNELL DAM & 25.9 & 8 & BR. OF HALLKILL RIVER & SUSSEX & FRANKLIN & LAKE GRINNELL ASSN. \\
\hline
\end{tabular}




\begin{tabular}{|c|c|c|c|c|c|c|}
\hline LAKE VALENTINE DAM & 55.0 & 12 & BRANCH OF PEQUEST RIVERS & SUSSEX & ANDOVER & FRANK KERSTNER \\
\hline NO NAME & 60.0 & 11 & CLOVE BROOK & SUSSEX & COLESVILLE & MR. AUTORE \\
\hline CLOVE RIVER DAM & 54.2 & 22 & CLOVE RIVER & SUSSEX & SUSSEX & SUSSEX BOROUGH \\
\hline HIGHLAND LAKE DAM & 6.8 & 22 & DOUBLE KILL & SUSSEX & HIGHLAND LAKE & HIGHLAND LAKES CTY \\
\hline LAKE OCQUITUNK DAM & 21.5 & 13 & FLAT BROOK & SUSSEX & WALLPACK & STATE OF NJ \\
\hline DEER TRAIL LAKE DAM & 50.0 & 30 & FRANKLIN POND CREEK & SUSSEX & FRANKLIN & \\
\hline REYNOLDS LAKE DAM & 61.0 & 13 & HEADWATERS PAULINS KILL & SUSSEX & LAFAYETTE & ISADORE GLAUBERMAN \\
\hline SENECA LAKE DAM & 60.0 & 11 & LUBBERS RUN & SUSSEX & LAKE LACKAWANNA & SENECA LAKE CLUB \\
\hline TOMAHAWK LAKE DAM & 70.0 & 13 & LUBBERS RUN & SUSSEX & LAKE LACKAWANMA & CHESTER WALLACE \\
\hline CANISTEAR RESERVOIR DAM & 33.9 & 55 & PACOCK BROOK & SUSSEX & STOCKHOLM & CITY OF NEWARK \\
\hline LAKE NEEPAULIN DAM & 65.3 & 10 & PAPAKATING CREEK & SUSSEX & SUSSEX & MEEPAUL IN COMMUNITY \\
\hline BALESVILLE DAM & 60.0 & 9 & PAULINS KILL & SUSSEX & BALESVILLE & \\
\hline SPARTA LAKE DAM & 48.0 & 7 & RUSSIA BROOK & SUSSEX & RUSSIA & SPARTA LAKE PROPERTY ASSOC. \\
\hline LAKE KATHRYN & 84.0 & 12 & SHALL BRANCH & SUSSEX & MIDDLEVILLE & \\
\hline STEENYKILL DAM & 37.0 & 25 & STEENY BROOK & SUSSEX & PORT JERVIS, NY & HIGH PT. PARK COMM. \\
\hline LAKE LENAPE DAM & 12.1 & 22 & TAR HILL BR. & SUSSEX & ANDOVER & LAKE LENAPE PROP. \\
\hline SUNSET LAKE DAM & 60.0 & 7 & TR-SPARTA GLEN & SUSSEX & SPARTA & MAPLE GUN CLUB \\
\hline KILLER DAM & 28.8 & 17 & TR. DELAWARE RIVER & SUSSEX & MILLVILLE & \\
\hline KOFFERLS POND DAM & 45.0 & 20 & TR. LUBBERS RUN & SUSSEX & ROSEVILLE & JAMES PROULX \\
\hline NO NAME & 45.0 & 17 & TR. PAPAKATING CREEK & SUSSEX & LEWISBURG & WESTGATE ASSOC. \\
\hline CLEARVIEW LAKE DAM & 53.0 & 13 & TR. PAULLINS KILL & SUSSEX & BALESVILLE & CENTRAL SUSSEX LAND CORP. \\
\hline No NAME & 80.0 & 8 & TR. PAULINS KILL & SUSSEX & SWART SHOOD & KENNETH BARISH \\
\hline THOMAS RICH & 64.0 & 13 & TR. PAULINS KILL & SUSSEX & PAULINS KILL & THOS. RICH \\
\hline LAKE ANNE DAM & 72.0 & 10 & TR. PAULINS KILL & SUSSEX & SWARTSWOOD & GALLANTI RESERVATION INC. \\
\hline LAWRENCE DAM & 80.0 & 10 & TR. PAULINS KILL & SUSSEX & LAFAYETTE & FARMSTEAD CC/B PHOEBUS \\
\hline BRADLEYS POND DAM & 46.0 & 18 & TR. PAULINS KILL & SUSSEX & PAULINS KILL & STANLEY PETER \\
\hline EMILIO PERONA DAM & 66.0 & 19 & TR. PEQUEST RIVER & susseX & LAKE LENAPE & EMIL PERONA \\
\hline FOREST LAKE DAM & 61.0 & 40 & TR. PEQUEST RIVER & SUSSEX & ANDOVER & FOREST LAKE CO. \\
\hline
\end{tabular}




\begin{tabular}{|c|c|c|c|c|c|c|}
\hline DENTON CLARK ESTATE DAM & 40.0 & 15 & TR. POCHUCK CREEK & SUSSEX & GLENWOOD & CAMP LOUEMMA \\
\hline HEMLOCK LAKE DAM & 22.0 & 38 & TR. REQUEST RIVER & SUSSEX & ANDOVER & WESTBY CORP. \\
\hline ACQUACKNONK LAKE DAM & 38.9 & 19 & TR. ROCKAWAY RIVER & SUSSEX & RUSSIA & YMCA PASSAIC \\
\hline ROCK ISLAND LAKE & 36.0 & 17 & TR. SPARTA GLEN & SUSSEX & SPARTA & MR. MONTANE/MR. AHRENS \\
\hline SHAY LAKE DAM & 64.0 & 24 & TR. TO FLAT BROOK & SUSSEX & WALLPACK & STATE OF NJ \\
\hline NO NAME \#19 & 55.0 & 10 & TR. TROUT BROOK & SUSSEX & STILLLATER & \\
\hline NO NAME DAM \#35 & 57.0 & 25 & TR. VAN CAMPENS BROOK & SUSSEX & MILLBROOK & BLUE MOUNTAIN COUNTRY CLUB \\
\hline BL.UE HERON LAKE DAM & 56.0 & 7 & TR. HALLKILL RIVER & SUSSEX & SPARTA & LAKELAND PROPERTY \\
\hline LAKE BURNBRAE DAM & 56.0 & 10 & TR. WALLKILL RIVER & SUSSEX & SPARTA & GLEN LAKE LODGE \\
\hline SLEEPY VALLEY DAM & 66.0 & 12 & TR. WALLKILL RIVER & SUSSEX & INDEPENDENCE CORNER & TALL TIMBER INC. \\
\hline LAKE PANORAMA DAM & 75.0 & 19 & TR. WALLKILL RIVER & SUSSEX & HANTAGE & LAKE PANORAMA ASSOC. \\
\hline FAWN LAKE & 55.0 & 21 & TR. WALLKILL RIVER & SUSSEX & OGDENBURG & \\
\hline MUD POND DAK & 60.0 & 8 & TR. WALLKILL RIVER & SUSSEX & HAMBURG & F. S. BURROWS \\
\hline NO NAME & 30.0 & 30 & TR. WALLKILLL RIVER & SUSSEX & WANTAGE & SCENIC LAKES COMMUNITY \\
\hline NO NAME & 60.0 & 13 & TR. HALLKILL RIVER & SUSSEX & WANTAGE & DAVE SIMMON \\
\hline KOHOUT LAKE DAM & 50.0 & 12 & TR. WAWAYANDA CREEK & SUSSEX & NEW MILFORD, NY & F. KOHOUT \\
\hline LAKE WANDA DAM & 2.3 & 6 & TR. MAHAYANDA CREEK & SUSSEX & WARHICK & KARL SCHWARTZ \\
\hline NO NAME \#26 & 96.0 & 12 & TR. WAWAYANDA CREEX & SUSSEX & WARWICK & \\
\hline BARRY LAKE \#1 DAM & 56.0 & 8 & IR. WAWAYANDA CREEK & SUSSEX & NEW MILFORD & BLUE RIDGE LAKES \\
\hline UPPER HIGHLAND DAM & 77.0 & 15 & TR. WAWAYANDA CREEK & SUSSEX & HIGHLAND LAKES & HIGHLAND LAKES CONM. ASSOC. \\
\hline NO NAME \#14 & 85.0 & 30 & TR. WEST BRANCH PAPAKATING CK & SUSSEX & MCCOY CORNER & WESTGATE ASSOC. \\
\hline LAKE PLYMOUTH DAM & 90.0 & 17 & TROUT BROOK & SUSSEX & MIDDLEVILLE & LAKE PLYMOUTH ASSOC. \\
\hline BLOODGOOD'S POND DAM & 55.9 & 13 & 4 STOW CR. UPPER BR. & UNION & CANTON & CITY OF RAHWAY \\
\hline SEELEY'S MILL. UPPER DAM & 12.1 & 20 & BLUE BR. & UNION & WATCHUNG RES. SCOTCH PLAIH & UNION CO. PARK COMM. \\
\hline SURPRISE LAKE DAM & 71.0 & 20 & BLUE BROOK & UNION & WATCHUNG RESERVATION MT. & UNION CYT PARKS COMM. \\
\hline RSINO LAKE DAM & 20.2 & 11 & ELIZABETH RIVER & UNION & ELIZABETH & UNION CO. PARK COMN. \\
\hline SHACKAMAXON DAM & $\mathbf{5 3 . 0}$ & 24 & LAMBERT'S RUN & UNION & SCOTCH PLAINS & SHACKAMAXON GOLF \& CITY \\
\hline
\end{tabular}




\begin{tabular}{|c|c|c|c|c|c|c|}
\hline LINDEN \#1 DAM & 86.0 & 14 & MORSES CREEK & UNION & CARTERET & EXXON CORP. \\
\hline ECHO LAKE (UPPER) DAM & 48.0 & 17 & NOMAHEGAN CREEK & UNION & KENILHORTH & COUNTY OF UNION PARK COMM. \\
\hline ECHO LAKE DAM & 68.0 & 12 & MORMAHIGGIN CREEK & UNION & CRANFORD & UNION CITY PARK \\
\hline BLOODGOOD'S POND DAM & 75.0 & 14 & RAHWAY RIVER & UNION & RAHWAY & UNION COUNTY PARKS COMM. \\
\hline JACKSONS POND DAM & 28.0 & 14 & RAHWAY RIVER & UNION & RAHWAY & CITY OF RAHWAY \\
\hline MILTON LAKE DAM & 30.0 & 10 & ROBINSONS BR. RAHWAY RIVER & UNION & RAHWAY & CTY. OF UNION PARK COMM. \\
\hline MIDDLESEX RESERVOIR DAM & 44.9 & 19 & ROBINSONS BROOK & UNION & RAHWAY & MIDDLESEX MATER CO. \\
\hline BRIANT PARK DAM & 51.0 & 25 & VAN UINKLE BROOK & UNION & SPRINGFIELD & UNION CTY PARKS COMM. \\
\hline LOCUST LAKE DAM & 5.6 & 20 & BEAVER BR. & WARREN & BELVIDERE & LOCUST LAKE CO. \\
\hline BASS LAKE DAM & 21.4 & 33 & BLAIRS CREEK & WARREN & BLAIRSTOWN & PRINCETON UNIVERSITY \\
\hline BLAIR LAKE DAM & 24.0 & 20 & BLAIRS CREEK & WARREN & BLAIRSTOWN & BLAIR ACADEMY \\
\hline SPRING VALLEY DAM & 55.0 & 13 & BRANCH BLAIRS CREEK & WARREN & BLAIRSTOWN & COTRONE \\
\hline NO NAME & 60.0 & 10 & BRANCH OF BEAVER BROOK & WARREN & HOPE & \\
\hline HACKETTSTOWN STORAGE RESEV & 68.0 & 38 & MINE BROOK & MARREN & BEATYESTOWN & HACKETTSTOWN, NJ \\
\hline MOUNTAIN LAKE DAM & 65.0 & 6 & MOUNTAIN LAKE BROOK & HARREN & BUTTZVILLE & LEWIS BUCHENAYER \\
\hline SAXTON FALLS DAM & 82.7 & 11 & MUSCONETCONG & WARREN & HACKETTSTOWN & STATE OF NJ \\
\hline NEWBURG MILL DAM & 35.0 & 10 & MUSCONETCONG RIVER & MARREN & BEATTYSTOWN & REICHOLD CHEMICAL CO. \\
\hline GRUENDYKE MILL DAM & 36.0 & 15 & MUSCONETCONG RIVER & HARREN & HACKETTSTOWN & 8. NOCELLA \\
\hline AUXILIARY RESERVOIR DAM & 51.0 & 20 & OFFSTREAM & MARREN & MALNUT VALLEY & CENTRAL JERSEY P\&L CO. \\
\hline FURNACE BROOK WATERSHED \#2 & 14.7 & 46 & OXFORO BROOK & HARREN & OXFORD & OXFORD TWP. \\
\hline PAULINA DAM & 17.2 & 13 & PAULINS KILL & WARREN & BLAIRSTOWN & BLAIRSTOWN HATER \& ELECTRIC \\
\hline PLEASANT VALLEY MILL DAM & 22.0 & 13 & POHATCONG CREEK & WARREN & BROADWAY & FRANK PERCAPPIO \\
\hline WILLEVER LAKE DAM & 61.0 & 8 & POHATCONG CREEK & WARREN & NEH VILLAGE & JC WILLEVER \\
\hline NO NAME DAM & 28.0 & 13 & POIPHANDUSING BROOK & WARREN & HAGEN & SAMUEL RACE \\
\hline No NAME & 24.0 & 30 & TR. BEAVER BROOK & WARREN & HOPE & JOHN LOIZ IIJ \\
\hline No NAME \#29 & 83.0 & 20 & TR. BLAIR CREEK & WARREN & BLAIRSTOWN & KOPYCINSKI \\
\hline ALAMUCHY POND DAM & 1.9 & 10 & TR. PEQUEST RIVER & WARREN & ALAMUCHY & NJDEP \\
\hline NO NAME \#3021 & 40.0 & 25 & TR. POPHANDUSING BROOK & WARREN & HAGEN & H. A. RACE \\
\hline
\end{tabular}


NO NAME \#3020

FRENCH LAKE DAM

POWER RESERVOIR DAM

- set print off $\begin{array}{lll}15.0 & 35 & \text { TR. POPHANDUSING BROOK }\end{array}$

TRIB. BEAR BROOK

$18.0 \quad 41 \quad$ YARDS CREEK
WARREN

MARREN

WARREN
HAGEN

JOHNSONBURG

HAINESBURG
M. E. RACE

JOHN MCDONOUGH

JCP\&L 